\title{
Almost periodic solutions for SICNNs with time-varying delays in the leakage terms
}

Bingwen Liu ${ }^{1}$ and Jianying Shao ${ }^{*}$

*Correspondence:
shaojianying2008@aliyun.com
${ }^{2}$ College of Mathematics, Physics
and Information Engineering,
Jiaxing University, Jiaxing, Zhejiang
314001, P.R. China
Full list of author information is
available at the end of the article

\begin{abstract}
This paper is concerned with the shunting inhibitory cellular neural networks (SICNNs) with time-varying delays in the leakage (or forgetting) terms. Under proper conditions, we employ a novel argument to establish a criterion on the global exponential stability of almost periodic solutions by using Lyapunov functional method and differential inequality techniques. We also provide numerical simulations to support the theoretical result.
\end{abstract}

Keywords: shunting inhibitory cellular neural networks; almost periodic solution; exponential stability; time-varying delay; leakage term

\section{Introduction}

It is well known that shunting inhibitory cellular neural networks (SICNNs) have been introduced as new cellular neural networks (CNNs) in Bouzerdout and Pinter in [1-3], which can be described by

$$
\begin{aligned}
x_{i j}^{\prime}(t)= & -a_{i j}(t) x_{i j}(t)-\sum_{C_{k l} \in N_{r}(i, j)} C_{i j}^{k l}(t) f\left(x_{k l}(t-\tau(t))\right) x_{i j}(t) \\
& -\sum_{C_{k l} \in N_{q}(i, j)} B_{i j}^{k l}(t) \int_{0}^{\infty} K_{i j}(u) g\left(x_{k l}(t-u)\right) d u x_{i j}(t)+L_{i j}(t), \\
& i=1,2, \ldots, m, j=1,2, \ldots, n,
\end{aligned}
$$

where $C_{i j}$ denotes the cell at the $(i, j)$ position of the lattice. The $r$-neighborhood $N_{r}(i, j)$ is given as

$$
N_{r}(i, j)=\left\{C_{k l}: \max (|k-i|,|l-j|) \leq r, 1 \leq k \leq m, 1 \leq l \leq n\right\},
$$

$N_{q}(i, j)$ is similarly specified. $x_{i j}$ is the activity of the cell $C_{i j}, L_{i j}(t)$ is the external input to $C_{i j}$, the function $a_{i j}(t)>0$ represents the passive decay rate of the cell activity, $C_{i j}^{k l}(t)$ and $B_{i j}^{k l}(t)$ are the connection or coupling strength of postsynaptic activity of the cell transmitted to the cell $C_{i j}$, and the activity functions $f(\cdot)$ and $g(\cdot)$ are continuous functions representing the output or firing rate of the cell $C_{k l}$, and $\tau(t) \geq 0$ corresponds to the transmission delay.

Recently, SICNNs have been extensively applied in psychophysics, speech, perception, robotics, adaptive pattern recognition, vision, and image processing. Hence, they have been the object of intensive analysis by numerous authors in recent years. In particular, 
there have been extensive results on the problem of the existence and stability of the equilibrium point, periodic and almost periodic solutions of SICNNs with time-varying delays in the literature. We refer the reader to [4-9] and the references cited therein. Obviously, the first term in each of the right side of (1.1) corresponds to a stabilizing negative feedback of the system, which acts instantaneously without time delay; these terms are variously known as 'forgetting' or leakage terms (see, for instance, Kosko [10], Haykin [11]). It is known from the literature on population dynamics and neural networks dynamics (see Gopalsamy [12]) that time delays in the stabilizing negative feedback terms will have a tendency to destabilize a system. Therefore, the authors of [13-19] dealt with the existence and stability of equilibrium and periodic solutions for neuron networks model involving leakage delays. However, to the best of our knowledge, few authors have considered the existence and exponential stability of almost periodic solutions of SICNNs with time-varying delays in the leakage terms. Motivated by the discussions above, in this paper, we consider the following SICNNs with time-varying leakage delays:

$$
\begin{aligned}
x_{i j}^{\prime}(t)= & -a_{i j}(t) x_{i j}\left(t-\eta_{i j}(t)\right)-\sum_{C_{k l} \in N_{r}(i, j)} C_{i j}^{k l}(t) f\left(x_{k l}(t-\tau(t))\right) x_{i j}(t) \\
& -\sum_{C_{k l} \in N_{q}(i, j)} B_{i j}^{k l}(t) \int_{0}^{\infty} K_{i j}(u) g\left(x_{k l}(t-u)\right) d u x_{i j}(t)+L_{i j}(t),
\end{aligned}
$$

where $i=1,2, \ldots, m, j=1,2, \ldots, n, a_{i j}: R \rightarrow(0+\infty), \eta_{i j}, \tau: R \rightarrow[0+\infty)$, and $L_{i j}, C_{i j}^{k l}, B_{i j}^{k l}:$ $R \rightarrow R$ are almost periodic functions, $\eta_{i}(t)$ and $\tau(t)$ denote the leakage delay and transmission delay, respectively, the delay kernels $K_{i j}:[0, \infty) \rightarrow[0, \infty)$ are continuous and integrable, and $\eta_{i j}^{\prime}$ is a bounded continuous function.

The main purpose of this paper is to give the conditions for the existence and exponential stability of the almost periodic solutions for system (1.2). By applying Lyapunov functional method and differential inequality techniques, we derive some new sufficient conditions ensuring the existence, uniqueness and exponential stability of the almost periodic solution for system (1.2), which are new and complement previously known results. Moreover, an example with numerical simulations is also provided to illustrate the effectiveness of our results.

Throughout this paper, for $i j \in J:=\{11,12, \ldots, 1 n, 21,22, \ldots, 2 n, \ldots, m 1, m 2, \ldots, m n\}$, from the theory of almost periodic functions in [20,21], it follows that for all $\epsilon>0$, it is possible to find a real number $l=l(\epsilon)>0$, for any interval with length $l(\epsilon)$, there exists a number $\delta=\delta(\epsilon)$ in this interval such that

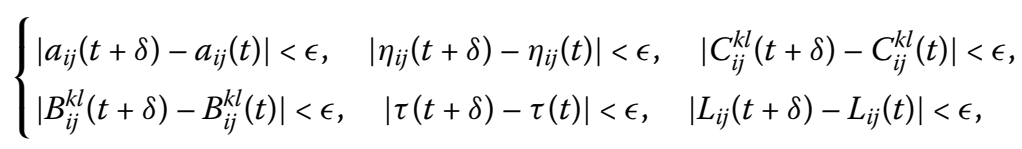

for all $t \in R, k l, i j \in J$.

We set

$$
\left\{x_{i j}(t)\right\}=\left(x_{11}(t), \ldots, x_{1 n}(t), \ldots, x_{i 1}(t), \ldots, x_{i n}(t), \ldots, x_{m 1}(t), \ldots, x_{m n}(t)\right) \in R^{m \times n}
$$


For $\forall x(t)=\left\{x_{i j}(t)\right\} \in R^{m \times n}$, we define the norm $\|x(t)\|=\max _{(i, j)}\left\{\left|x_{i j}(t)\right|\right\}$. For the convenience, we shall introduce the notations

$$
h^{+}=\sup _{t \in R}|h(t)|
$$

where $h(t)$ is a bounded continuous function.

We also make the following assumptions.

( $\left.\mathrm{T}_{1}\right)$ There exist constants $M_{f}, M_{g}, \mu_{f}$ and $\mu_{g}$ such that

$$
\begin{array}{ll}
|f(u)-f(v)| \leq \mu_{f}|u-v|, & |f(u)| \leq M_{f}, \\
|g(u)-g(v)| \leq \mu_{g}|u-v|, & |g(u)| \leq M_{g}, \quad \text { for all } u, v \in R .
\end{array}
$$

$\left(\mathrm{T}_{2}\right)$ For $i j \in J, a_{i j}^{+} \eta_{i j}^{+}<1$,

$$
\begin{aligned}
\delta_{i j}(t)= & \left\{a_{i j}(t)\left(1-2 a_{i j}^{+} \eta_{i j}^{+}\right)-\left|a_{i j}(t)-\left(1-\eta_{i j}^{\prime}(t)\right) a_{i j}\left(t-\eta_{i j}(t)\right)\right|\right. \\
& -\sum_{C_{k l} \in N_{r}(i, j)}\left|C_{i j}^{k l}(t)\right| M_{f} \\
& \left.-\sum_{C_{k l} \in N_{q}(i, j)}\left|B_{i j}^{k l}(t)\right| \int_{0}^{\infty}\left|K_{i j}(u)\right| d u M_{g}\right\} \frac{1}{1-a_{i j}^{+} \eta_{i j}^{+}} \\
> & 0,
\end{aligned}
$$

and there exist positive constants $\eta>0$ and $\lambda$ such that

$$
\lambda<a_{i j}(t), \quad \int_{0}^{\infty} e^{\lambda u}\left|K_{i j}(u)\right| d u<+\infty
$$

and

$$
\begin{aligned}
& -\left[\left(a_{i j}(t)-\lambda\right)\left(1-2 a_{i j}^{+} \eta_{i j}^{+}\right)\right. \\
& \left.-\left|a_{i j}(t) e^{\lambda \eta_{i j}(t)}-\left(1-\eta_{i j}^{\prime}(t)\right) a_{i j}\left(t-\eta_{i j}(t)\right)\right|\right] \frac{1}{1-a_{i j}^{+} \eta_{i j}^{+}} \\
& +\sum_{C_{k l} \in N_{r}(i, j)}\left|C_{i j}^{k l}(t)\right| \mu_{f} e^{\lambda \tau(t)} \frac{1}{1-a_{k l}^{+} \eta_{k l}^{+}} \frac{1}{1-a_{i j}^{+} \eta_{i j}^{+}}\left(\frac{L_{i j}}{\delta_{i j}}\right)^{+} \\
& +\sum_{C_{k l} \in N_{r}(i, j)}\left|C_{i j}^{k l}(t)\right| M_{f} \frac{1}{1-a_{i j}^{+} \eta_{i j}^{+}} \\
& +\sum_{C_{k l} \in N_{q}(i, j)}\left|B_{i j}^{k l}(t)\right| \int_{0}^{\infty} e^{\lambda u}\left|K_{i j}(u)\right| d u \mu_{g} \frac{1}{1-a_{k l}^{+} \eta_{k l}^{+}} \frac{1}{1-a_{i j}^{+} \eta_{i j}^{+}}\left(\frac{L_{i j}}{\delta_{i j}}\right)^{+} \\
& +\sum_{C_{k l} \in N_{q}(i, j)}\left|B_{i j}^{k l}(t)\right| \int_{0}^{\infty}\left|K_{i j}(u)\right| d u M_{g} \frac{1}{1-a_{i j}^{+} \eta_{i j}^{+}} \\
& <-\eta, \quad \text { for all } t \geq 0 \text {. }
\end{aligned}
$$


The initial conditions associated with system (1.2) are of the form

$$
x_{i j}(s)=\varphi_{i j}(s), \quad s \in(-\infty, 0], i j \in J
$$

where $\varphi_{i j}(\cdot)$ denotes a real-valued bounded continuous function defined on $(-\infty, 0]$.

Definition 1.1 (See $[20,21]$ ) Let $u(t): R \longrightarrow R^{m \times n}$ be continuous in $t . u(t)$ is said to be almost periodic on $R$ if for any $\varepsilon>0$, the set $T(u, \varepsilon)=\{\delta:\|u(t+\delta)-u(t)\|<\varepsilon, \forall t \in R\}$ is relatively dense, i.e., for any $\varepsilon>0$, it is possible to find a real number $l=l(\varepsilon)>0$, for any interval with length $l(\varepsilon)$, there exists a number $\delta=\delta(\varepsilon)$ in this interval such that $\| u(t+\delta)-$ $u(t) \|<\varepsilon$ for all $t \in R$.

\section{Preliminary results}

The following lemmas will be useful to prove our main results in Section 3.

Lemma 2.1 Let $\left(T_{1}\right)$ and $\left(T_{2}\right)$ hold. Suppose that $x(t)=\left\{x_{i j}(t)\right\}$ is a solution of system (1.2) with initial conditions

$$
x_{i j}(s)=\varphi_{i j}(s), \quad\left|\varphi_{i j}(t)-\int_{t-\eta_{i j}(t)}^{t} a_{i j}(s) \varphi_{i j}(s) d s\right|<\left(\frac{L_{i j}}{\delta_{i j}}\right)^{+}, \quad s, t \in(-\infty, 0], i j \in J .
$$

Then

$$
\left|x_{i j}(t)-\int_{t-\eta_{i j}(t)}^{t} a_{i j}(s) x_{i j}(s) d s\right| \leq\left(\frac{L_{i j}}{\delta_{i j}}\right)^{+}, \text {for all } t \geq 0, i j \in J
$$

and

$$
\left|x_{i j}(t)\right| \leq \frac{1}{1-a_{i j}^{+} \eta_{i j}^{+}}\left(\frac{L_{i j}}{\delta_{i j}}\right)^{+}, \text {for all } t \geq 0, i j \in J \text {. }
$$

Proof Assume, by way of contradiction, that (2.2) does not hold. Then, there exist $i j \in \Lambda$, $\gamma>\left(\frac{L_{i j}}{\delta_{i j}}\right)^{+}$and $t_{*}>0$ such that

$$
\left|X_{i j}\left(t_{*}\right)\right|=\gamma \quad \text { and } \quad\left|X_{i j}(t)\right|<\gamma \quad \text { for all } t \in\left(-\infty, t_{*}\right) \text {, }
$$

where

$$
X_{i j}(t)=x_{i j}(t)-\int_{t-\eta_{i j}(t)}^{t} a_{i j}(s) x_{i j}(s) d s \text {. }
$$

It follows that

$$
\begin{aligned}
\left|x_{i j}(t)\right| & \leq\left|x_{i j}(t)-\int_{t-\eta_{i j}(t)}^{t} a_{i j}(s) x_{i j}(s) d s\right|+\left|\int_{t-\eta_{i j}(t)}^{t} a_{i j}(s) x_{i j}(s) d s\right| \\
& \leq \gamma+a_{i j}^{+} \eta_{i j}^{+} \sup _{s \in\left(-\infty, t_{*}\right]}\left|x_{i j}(s)\right|, \quad \text { for all } t \in\left(-\infty, t_{*}\right] .
\end{aligned}
$$


Consequently, in view of (2.5) and the fact $a_{i j}^{+} \eta_{i j}^{+}<1(i j \in J)$, we have

$$
\begin{aligned}
\left|x_{i j}(t)\right| & \leq \sup _{s \in\left(-\infty, t_{*}\right]}\left|x_{i j}(s)\right| \\
& \leq \frac{1}{1-a_{i j}^{+} \eta_{i j}^{+}} \gamma, \quad \text { for all } t \in\left(-\infty, t_{*}\right] .
\end{aligned}
$$

From system (1.2), we derive

$$
\begin{aligned}
\frac{d}{d t}\left(x_{i j}(t)-\int_{t-\eta_{i j}(t)}^{t} a_{i j}(s) x_{i j}(s) d s\right) & \\
= & x_{i j}^{\prime}(t)-\left[a_{i j}(t) x_{i j}(t)-\left(1-\eta_{i j}^{\prime}(t)\right) a_{i j}\left(t-\eta_{i j}(t)\right) x_{i j}\left(t-\eta_{i j}(t)\right)\right] \\
= & -\left[a_{i j}(t) x_{i j}(t)-\left(1-\eta_{i j}^{\prime}(t)\right) a_{i j}\left(t-\eta_{i j}(t)\right) x_{i j}\left(t-\eta_{i j}(t)\right)\right] \\
& +\left[-a_{i j}(t) x_{i}\left(t-\eta_{i j}(t)\right)-\sum_{C_{k l} \in N_{r}(i, j)} C_{i j}^{k l}(t) f\left(x_{k l}(t-\tau(t))\right) x_{i j}(t)\right. \\
& \left.-\sum_{C_{k l} \in N_{q}(i, j)} B_{i j}^{k l}(t) \int_{0}^{\infty} K_{i j}(u) g\left(x_{k l}(t-u)\right) d u x_{i j}(t)+L_{i j}(t)\right] \\
= & -a_{i j}(t) x_{i j}(t)-\left[a_{i j}(t)-\left(1-\eta_{i j}^{\prime}(t)\right) a_{i j}\left(t-\eta_{i j}(t)\right)\right] x_{i j}\left(t-\eta_{i j}(t)\right) \\
& -\sum_{C_{k l} \in N_{r}(i, j)} C_{i j}^{k l}(t) f\left(x_{k l}(t-\tau(t))\right) x_{i j}(t) \\
& -\sum_{C_{k l} \in N_{q}(i, j)} B_{i j}^{k l}(t) \int_{0}^{\infty} K_{i j}(u) g\left(x_{k l}(t-u)\right) d u x_{i j}(t)+L_{i j}(t) \\
= & -a_{i j}(t)\left(x_{i j}(t)-\int_{t-\eta_{i j}(t)}^{t} a_{i j}(s) x_{i j}(s) d s\right)-a_{i j}(t) \int_{t-\eta_{i j}(t)}^{t} a_{i j}(s) x_{i j}(s) d s \\
& -\left[a_{i j}(t)-\left(1-\eta_{i j}^{\prime}(t)\right) a_{i j}\left(t-\eta_{i j}(t)\right)\right] x_{i j}\left(t-\eta_{i}(t)\right) \\
& -\sum_{C_{k l} \in N_{r}(i, j)} C_{i j}^{k l}(t) f\left(x_{k l}(t-\tau(t))\right) x_{i j}(t) \\
& -\sum_{C_{k l} \in N_{q}(i, j)} B_{i j}^{k l}(t) \int_{0}^{\infty} K_{i j}(u) g\left(x_{k l}(t-u)\right) d u x_{i j}(t)+L_{i j}(t), \quad i j \in J . \\
& \sum_{i j}(t)
\end{aligned}
$$

Calculating the upper left derivative of $\left|X_{i j}(t)\right|$, together with (2.4), (2.6), (2.7), ( $\left.\mathrm{T}_{1}\right)$ and $\left(\mathrm{T}_{2}\right)$, we obtain

$$
\begin{aligned}
0 \leq & D^{-}\left|X_{i j}\left(t_{*}\right)\right| \\
\leq & -a_{i j}\left(t_{*}\right)\left|X_{i j}\left(t_{*}\right)\right|+\mid-a_{i j}\left(t_{*}\right) \int_{t_{*}-\eta_{i j}\left(t_{*}\right)}^{t_{*}} a_{i j}(s) x_{i j}(s) d s \\
& -\left[a_{i j}\left(t_{*}\right)-\left(1-\eta_{i j}^{\prime}\left(t_{*}\right)\right) a_{i j}\left(t_{*}-\eta_{i j}\left(t_{*}\right)\right)\right] x_{i j}\left(t_{*}-\eta_{i j}\left(t_{*}\right)\right) \\
& -\sum_{C_{k l} \in N_{r}(i, j)} C_{i j}^{k l}\left(t_{*}\right) f\left(x_{k l}\left(t_{*}-\tau\left(t_{*}\right)\right)\right) x_{i j}\left(t_{*}\right) \\
& -\sum_{C_{k l} \in N_{q}(i, j)} B_{i j}^{k l}\left(t_{*}\right) \int_{0}^{\infty} K_{i j}(u) g\left(x_{k l}\left(t_{*}-u\right)\right) d u x_{i j}\left(t_{*}\right)+L_{i j}\left(t_{*}\right) \mid
\end{aligned}
$$




$$
\begin{aligned}
& \leq-a_{i j}\left(t_{*}\right)\left|X_{i j}\left(t_{*}\right)\right|+a_{i j}\left(t_{*}\right) \int_{t_{*}-\eta_{i j}\left(t_{*}\right)}^{t_{*}} a_{i j}^{+}\left|x_{i j}(s)\right| d s \\
& +\left|a_{i j}\left(t_{*}\right)-\left(1-\eta_{i j}^{\prime}\left(t_{*}\right)\right) a_{i j}\left(t_{*}-\eta_{i j}\left(t_{*}\right)\right)\right|\left|x_{i j}\left(t_{*}-\eta_{i j}\left(t_{*}\right)\right)\right| \\
& +\sum_{C_{k l} \in N_{r}(i, j)}\left|C_{i j}^{k l}\left(t_{*}\right)\right|\left|f\left(x_{k l}\left(t_{*}-\tau\left(t_{*}\right)\right)\right)\right|\left|x_{i j}\left(t_{*}\right)\right| \\
& +\sum_{C_{k l} \in N_{q}(i, j)}\left|B_{i j}^{k l}\left(t_{*}\right)\right| \int_{0}^{\infty}\left|K_{i j}(u)\right|\left|g\left(x_{k l}\left(t_{*}-u\right)\right)\right| d u\left|x_{i j}\left(t_{*}\right)\right|+\left|L_{i j}\left(t_{*}\right)\right| \\
& \leq-a_{i j}\left(t_{*}\right)\left|X_{i j}\left(t_{*}\right)\right|+a_{i j}\left(t_{*}\right) \int_{t_{*}-\eta_{i j}\left(t_{*}\right)}^{t_{*}} a_{i j}^{+}\left|x_{i j}(s)\right| d s \\
& +\left|a_{i j}\left(t_{*}\right)-\left(1-\eta_{i j}^{\prime}\left(t_{*}\right)\right) a_{i j}\left(t_{*}-\eta_{i j}\left(t_{*}\right)\right)\right|\left|x_{i j}\left(t_{*}-\eta_{i j}\left(t_{*}\right)\right)\right| \\
& +\sum_{C_{k l} \in N_{r}(i, j)}\left|C_{i j}^{k l}\left(t_{*}\right)\right| M_{f}\left|x_{i j}\left(t_{*}\right)\right| \\
& +\sum_{C_{k l} \in N_{q}(i, j)}\left|B_{i j}^{k l}\left(t_{*}\right)\right| \int_{0}^{\infty}\left|K_{i j}(u)\right| d u M_{g}\left|x_{i j}\left(t_{*}\right)\right|+\left|L_{i j}\left(t_{*}\right)\right| \\
& \leq-a_{i j}\left(t_{*}\right) \gamma+a_{i j}\left(t_{*}\right) a_{i j}^{+} \eta_{i j}^{+} \frac{1}{1-a_{i j}^{+} \eta_{i j}^{+}} \gamma \\
& +\left|a_{i j}\left(t_{*}\right)-\left(1-\eta_{i j}^{\prime}\left(t_{*}\right)\right) a_{i j}\left(t_{*}-\eta_{i j}\left(t_{*}\right)\right)\right| \frac{\gamma}{1-a_{i j}^{+} \eta_{i j}^{+}} \\
& +\sum_{C_{k l} \in N_{r}(i, j)}\left|C_{i j}^{k l}\left(t_{*}\right)\right| M_{f} \frac{1}{1-a_{i j}^{+} \eta_{i j}^{+}} \gamma \\
& +\sum_{C_{k l} \in N_{q}(i, j)}\left|B_{i j}^{k l}\left(t_{*}\right)\right| \int_{0}^{\infty}\left|K_{i j}(u)\right| d u M_{g} \frac{1}{1-a_{i j}^{+} \eta_{i j}^{+}} \gamma+\left|L_{i j}\left(t_{*}\right)\right| \\
& =\left\{-\left[a_{i j}\left(t_{*}\right)\left(1-2 a_{i j}^{+} \eta_{i j}^{+}\right)-\left|a_{i j}\left(t_{*}\right)-\left(1-\eta_{i j}^{\prime}\left(t_{*}\right)\right) a_{i j}\left(t_{*}-\eta_{i j}\left(t_{*}\right)\right)\right|\right]\right. \\
& +\sum_{C_{k l} \in N_{r}(i, j)}\left|C_{i j}^{k l}\left(t_{*}\right)\right| M_{f} \\
& \left.+\sum_{C_{k l} \in N_{q}(i, j)}\left|B_{i j}^{k l}\left(t_{*}\right)\right| \int_{0}^{\infty}\left|K_{i j}(u)\right| d u M_{g}\right\} \frac{1}{1-a_{i j}^{+} \eta_{i j}^{+}} \gamma+\left|L_{i j}\left(t_{*}\right)\right| \\
& \leq-\delta_{i j}\left(t_{*}\right)\left[\gamma-\left(\frac{L_{i j}}{\delta_{i j}}\right)^{+}\right] \\
& <0 \text {. }
\end{aligned}
$$

It is a contradiction, and it shows that (2.2) holds. Then, using a similar argument as in the proof of (2.5) and (2.6), we can show that (2.3) holds. The proof of Lemma 2.1 is now completed.

Remark 2.1 In view of the boundedness of this solution, from the theory of functional differential equations with infinite delay in [22], it follows that the solution of system (1.2) with initial conditions $(2.1)$ can be defined on $[0,+\infty)$. 
Lemma 2.2 Suppose that $\left(\mathrm{T}_{1}\right)$ and $\left(\mathrm{T}_{2}\right)$ hold. Moreover, assume that $x(t)=\left\{x_{i j}(t)\right\}$ is a solution of system (1.2) with initial function $\varphi_{i j}(\cdot)$ satisfying $(2.1)$, and $\varphi_{i j}^{\prime}(\cdot)$ is bounded continuous on $(-\infty, 0]$. Then for any $\epsilon>0$, there exists $l=l(\epsilon)>0$, such that every interval $[\alpha, \alpha+l]$ contains at least one number $\delta$, for which there exists $N>0$ satisfying

$$
\|x(t+\delta)-x(t)\| \leq \epsilon, \quad \text { for all } t>N
$$

Proof For $i j \in J$, set

$$
\begin{aligned}
\epsilon_{i j}(\delta, t)= & -a_{i j}(t)\left[x_{i j}\left(t+\delta-\eta_{i j}(t+\delta)\right)-x_{i j}\left(t-\eta_{i j}(t)+\delta\right)\right] \\
& -\left[a_{i j}(t+\delta)-a_{i j}(t)\right] x_{i j}\left(t+\delta-\eta_{i j}(t+\delta)\right) \\
& -\sum_{C_{k l} \in N_{r}(i, j)}\left[C_{i j}^{k l}(t+\delta)-C_{i j}^{k l}(t)\right] f\left(x_{k l}(t-\tau(t+\delta)+\delta)\right) x_{i j}(t+\delta) \\
& -\sum_{C_{k l} \in N_{r}(i, j)} C_{i j}^{k l}(t)\left[f\left(x_{k l}(t-\tau(t+\delta)+\delta)\right)-f\left(x_{k l}(t-\tau(t)+\delta)\right)\right] x_{i j}(t+\delta) \\
& -\sum_{C_{k l} \in N_{q}(i, j)}\left[B_{i j}^{k l}(t+\delta)-B_{i j}^{k l}(t)\right] \int_{0}^{\infty} K_{i j}(u) g\left(x_{k l}(t+\delta-u)\right) d u x_{i j}(t+\delta) \\
& +\left[L_{i j}(t+\delta)-L_{i j}(t)\right] .
\end{aligned}
$$

By Lemma 2.1, the solution $x(t)=\left\{x_{i j}(t)\right\}$ is bounded and

$$
\left|x_{i j}(t)\right| \leq \frac{1}{1-a_{i j}^{+} \eta_{i j}^{+}}\left(\frac{L_{i j}}{\delta_{i j}}\right)^{+} \quad \text { for all } t \in[0,+\infty), i j \in J
$$

Thus, the right side of (1.2) is also bounded, which implies that $x(t)$ is uniformly continuous on $R$. From (1.3), for any $\epsilon>0$, there exists $l=l(\epsilon)>0$, such that every interval $[\alpha, \alpha+l]$, $\alpha \in R$, contains a $\delta$, for which

$$
\left|\epsilon_{i j}(\delta, t)\right| \leq \frac{1}{2} \eta \min _{\tilde{i} j \in J}\left\{1-a_{i j}^{+} \eta_{i j}^{+}\right\} \epsilon, \quad \text { where } i j \in J, t \in R \text {. }
$$

Let $N_{0} \geq 0$ be sufficiently large such that $t+\delta \geq 0$ for $t \geq N_{0}$, and denote $u_{i j}(t)=x_{i j}(t+$ $\delta)-x_{i j}(t)$. We obtain

$$
\begin{aligned}
\frac{d u_{i j}(t)}{d t}= & -a_{i j}(t) u_{i j}\left(t-\eta_{i j}(t)\right)-\sum_{C_{k l} \in N_{r}(i, j)} C_{i j}^{k l}(t)\left(f\left(x_{k l}(t-\tau(t)+\delta)\right)\right. \\
& \left.-f\left(x_{k l}(t-\tau(t))\right)\right) x_{i j}(t+\delta)-\sum_{C_{k l} \in N_{r}(i, j)} C_{i j}^{k l}(t) f\left(x_{k l}(t-\tau(t))\right) \\
& \cdot\left(x_{i j}(t+\delta)-x_{i j}(t)\right)-\sum_{C_{k l} \in N_{q}(i, j)} B_{i j}^{k l}(t) \int_{0}^{\infty} K_{i j}(u)\left(g\left(x_{k l}(t+\delta-u)\right)\right. \\
& \left.-g\left(x_{k l}(t-u)\right)\right) d u x_{i j}(t+\delta)-\sum_{C_{k l} \in N_{q}(i, j)} B_{i j}^{k l}(t) \int_{0}^{\infty} K_{i j}(u) g\left(x_{k l}(t-u)\right) d u \\
& \cdot\left(x_{i j}(t+\delta)-x_{i j}(t)\right)+\epsilon_{i j}(\delta, t), \quad \text { for all } t \geq N_{0}, i j \in J,
\end{aligned}
$$


which yields

$$
\begin{aligned}
& \frac{d}{d t}\left(e^{\lambda t} u_{i j}(t)-\int_{t-\eta_{i j}(t)}^{t} a_{i j}(s) e^{\lambda s} u_{i j}(s) d s\right) \\
& =\lambda e^{\lambda t} u_{i j}(t)+e^{\lambda t} u_{i j}^{\prime}(t) \\
& -\left[a_{i j}(t) e^{\lambda t} u_{i j}(t)-\left(1-\eta_{i j}^{\prime}(t)\right) c_{i j}\left(t-\eta_{i j}(t)\right) e^{\lambda\left(t-\eta_{i j}(t)\right)} u_{i j}\left(t-\eta_{i j}(t)\right)\right] \\
& =\lambda e^{\lambda t} u_{i j}(t)-\left[a_{i j}(t) e^{\lambda t} u_{i j}(t)-\left(1-\eta_{i j}^{\prime}(t)\right) a_{i j}\left(t-\eta_{i j}(t)\right) e^{\lambda\left(t-\eta_{i j}(t)\right)} u_{i j}\left(t-\eta_{i j}(t)\right)\right] \\
& +e^{\lambda t}\left\{-a_{i j}(t) u_{i j}\left(t-\eta_{i j}(t)\right)-\sum_{C_{k l} \in N_{r}(i, j)} C_{i j}^{k l}(t)\left(f\left(x_{k l}(t-\tau(t)+\delta)\right)\right.\right. \\
& \left.-f\left(x_{k l}(t-\tau(t))\right)\right) x_{i j}(t+\delta)-\sum_{C_{k l} \in N_{r}(i, j)} C_{i j}^{k l}(t) f\left(x_{k l}(t-\tau(t))\right) \\
& \cdot\left(x_{i j}(t+\delta)-x_{i j}(t)\right)-\sum_{C_{k l} \in N_{q}(i, j)} B_{i j}^{k l}(t) \int_{0}^{\infty} K_{i j}(u)\left(g\left(x_{k l}(t+\delta-u)\right)\right. \\
& \left.-g\left(x_{k l}(t-u)\right)\right) d u x_{i j}(t+\delta)-\sum_{C_{k l} \in N_{q}(i, j)} B_{i j}^{k l}(t) \int_{0}^{\infty} K_{i j}(u) g\left(x_{k l}(t-u)\right) d u \\
& \left.\cdot\left(x_{i j}(t+\delta)-x_{i j}(t)\right)+\epsilon_{i j}(\delta, t)\right\} \\
& =-\left(a_{i j}(t)-\lambda\right) e^{\lambda t} u_{i j}(t) \\
& -\left[a_{i j}(t)-\left(1-\eta_{i j}^{\prime}(t)\right) a_{i j}\left(t-\eta_{i j}(t)\right) e^{-\lambda \eta_{i j}(t)}\right] e^{\lambda t} u_{i j}\left(t-\eta_{i j}(t)\right) \\
& +e^{\lambda t}\left\{-\sum_{C_{k l} \in N_{r}(i, j)} C_{i j}^{k l}(t)\left(f\left(x_{k l}(t-\tau(t)+\delta)\right)\right.\right. \\
& \left.-f\left(x_{k l}(t-\tau(t))\right)\right) x_{i j}(t+\delta)-\sum_{C_{k l} \in N_{r}(i, j)} C_{i j}^{k l}(t) f\left(x_{k l}(t-\tau(t))\right) \\
& \cdot\left(x_{i j}(t+\delta)-x_{i j}(t)\right)-\sum_{C_{k l} \in N_{q}(i, j)} B_{i j}^{k l}(t) \int_{0}^{\infty} K_{i j}(u)\left(g\left(x_{k l}(t+\delta-u)\right)\right. \\
& \left.-g\left(x_{k l}(t-u)\right)\right) d u x_{i j}(t+\delta)-\sum_{C_{k l} \in N_{q}(i, j)} B_{i j}^{k l}(t) \int_{0}^{\infty} K_{i j}(u) g\left(x_{k l}(t-u)\right) d u \\
& \left.\cdot\left(x_{i j}(t+\delta)-x_{i j}(t)\right)+\epsilon_{i j}(\delta, t)\right\} \\
& =-\left(a_{i j}(t)-\lambda\right)\left(e^{\lambda t} a_{i j}(t)-\int_{t-\eta_{i j}(t)}^{t} a_{i j}(s) e^{\lambda s} u_{i j}(s) d s\right) \\
& -\left(a_{i j}(t)-\lambda\right) \int_{t-\eta_{i j}(t)}^{t} a_{i j}(s) e^{\lambda s} u_{i j}(s) d s \\
& -\left[a_{i j}(t)-\left(1-\eta_{i j}^{\prime}(t)\right) a_{i j}\left(t-\eta_{i j}(t)\right) e^{-\lambda \eta_{i j}(t)}\right] e^{\lambda t} u_{i j}\left(t-\eta_{i j}(t)\right) \\
& +e^{\lambda t}\left\{-\sum_{C_{k l} \in N_{r}(i, j)} C_{i j}^{k l}(t)\left(f\left(x_{k l}(t-\tau(t)+\delta)\right)\right.\right. \\
& \left.-f\left(x_{k l}(t-\tau(t))\right)\right) x_{i j}(t+\delta)-\sum_{C_{k l} \in N_{r}(i, j)} C_{i j}^{k l}(t) f\left(x_{k l}(t-\tau(t))\right)
\end{aligned}
$$




$$
\begin{aligned}
& \cdot\left(x_{i j}(t+\delta)-x_{i j}(t)\right)-\sum_{C_{k l} \in N_{q}(i, j)} B_{i j}^{k l}(t) \int_{0}^{\infty} K_{i j}(u)\left(g\left(x_{k l}(t+\delta-u)\right)\right. \\
& \left.-g\left(x_{k l}(t-u)\right)\right) d u x_{i j}(t+\delta)-\sum_{C_{k l} \in N_{q}(i, j)} B_{i j}^{k l}(t) \int_{0}^{\infty} K_{i j}(u) g\left(x_{k l}(t-u)\right) d u \\
& \left.\cdot\left(x_{i j}(t+\delta)-x_{i j}(t)\right)+\epsilon_{i j}(\delta, t)\right\}, \quad \text { for all } t \geq N_{0}, i j \in J .
\end{aligned}
$$

Set

$$
U(t)=\left\{U_{i j}(t)\right\}
$$

where

$$
U_{i j}(t)=e^{\lambda t} u_{i j}(t)-\int_{t-\eta_{i j}(t)}^{t} a_{i j}(s) e^{\lambda s} u_{i j}(s) d s, \quad i j \in J
$$

Let $(i j)_{t}$ be such an index that

$$
\left|U_{(i j) t}(t)\right|=\|U(t)\|
$$

Calculating the upper left derivative of $\left|U_{(i)_{s}}(s)\right|$ along (2.11), we have

$$
\begin{aligned}
D^{-}( & \left.\left|U_{(i j)_{s}}(s)\right|\right)\left.\right|_{s=t} \\
\leq & -\left(a_{(i j)_{t}}(t)-\lambda\right)\left|U_{(i j)_{t}}(t)\right|+\mid-\left(a_{(i j)_{t}}(t)-\lambda\right) \int_{t-\eta_{(i j)_{t}}(t)}^{t} a_{(i j)_{t}}(s) e^{\lambda s} u_{(i j)_{t}}(s) d s \\
& -\left[a_{(i j)_{t}}(t)-\left(1-\eta_{(i j)_{t}}^{\prime}(t)\right) a_{(i j)_{t}}\left(t-\eta_{(i j)_{t}}(t)\right) e^{-\lambda \eta_{(i j)_{t}}}(t)\right] e^{\lambda t} u_{(i j)_{t}}\left(t-\eta_{(i j)_{t}}(t)\right) \\
& +e^{\lambda t}\left\{-\sum_{C_{k l} \in N_{r}(i, j)_{t}} C_{(i j)_{t}}^{k l}(t)\left(f\left(x_{k l}(t-\tau(t)+\delta)\right)\right.\right. \\
& \left.-f\left(x_{k l}(t-\tau(t))\right)\right) x_{(i j)_{t}}(t+\delta)-\sum_{C_{k l} \in N_{r}(i, j)_{t}} C_{(i j)_{t}}^{k l}(t) f\left(x_{k l}(t-\tau(t))\right) \\
& \cdot\left(x_{(i j)_{t}}(t+\delta)-x_{(i j)_{t}}(t)\right)-\sum_{C_{k l} \in N_{q}(i, j)_{t}} B_{(i j)_{t}}^{k l}(t) \int_{0}^{\infty} K_{(i j)_{t}}(u)\left(g\left(x_{k l}(t+\delta-u)\right)\right. \\
& \left.-g\left(x_{k l}(t-u)\right)\right) d u x_{(i j)_{t}}(t+\delta)-\sum_{C_{k l} \in N_{q}(i, j)_{t}} B_{(i j)_{t}}^{k l}(t) \int_{0}^{\infty} K_{(i j)_{t}}(u) g\left(x_{k l}(t-u)\right) d u \\
& \left.\cdot\left(x_{(i j)_{t}}(t+\delta)-x_{(i j)_{t}}(t)\right)+\epsilon_{(i j)_{t}}(\delta, t)\right\}|\cdot|
\end{aligned}
$$

Let

$$
M(t)=\sup _{s \leq t}\{\|U(s)\|\} .
$$


It is obvious that $\|U(t)\| \leq M(t)$, and $M(t)$ is non-decreasing. In particular,

$$
\begin{aligned}
e^{\lambda \rho}\left|u_{i j}(\rho)\right| & \leq\left|e^{\lambda \rho} u_{i j}(\rho)-\int_{\rho-\eta_{i j}(\rho)}^{\rho} a_{i j}(s) e^{\lambda s} u_{i j}(s) d s\right|+\left|\int_{\rho-\eta_{i j}(\rho)}^{\rho} a_{i j}(s) e^{\lambda s} u_{i j}(s) d s\right| \\
& \leq M(t)+a_{i j}^{+} \eta_{i j}^{+} \sup _{\theta \in(-\infty, t]} e^{\lambda \theta}\left|u_{i j}(\theta)\right|, \quad \text { for all } t \geq \rho, i j \in J .
\end{aligned}
$$

Consequently, in view of (2.15) and the fact $a_{i j}^{+} \eta_{i j}^{+}<1(i j \in J)$, we have

$$
e^{\lambda s}\left|u_{i j}(s)\right| \leq \sup _{\theta \in(-\infty, t]} e^{\lambda \theta}\left|u_{i j}(\theta)\right| \leq \frac{M(t)}{1-a_{i j}^{+} \eta_{i j}^{+}}, \quad \text { where } s \in(-\infty, t], i j \in J
$$

Now, we consider two cases.

Case (i). If

$$
M(t)>\|U(t)\| \quad \text { for all } t \geq N_{0}
$$

Then, we claim that

$$
M(t) \equiv M\left(N_{0}\right) \text { is a constant for all } t \geq N_{0} .
$$

Assume, by way of contradiction, that (2.18) does not hold. Then, there exists $t_{1}>N_{0}$ such that $M\left(t_{1}\right)>M\left(N_{0}\right)$. Since

$$
\|U(t)\| \leq M\left(N_{0}\right) \quad \text { for all } t \leq N_{0} .
$$

There must exist $\beta \in\left(N_{0}, t_{1}\right)$ such that

$$
\|U(\beta)\|=M\left(t_{1}\right) \geq M(\beta),
$$

which contradicts (2.17). This contradiction implies that (2.18) holds. It follows from (2.16) that there exists $t_{2}>N_{0}$ such that

$$
\|u(t)\|=\max _{i j \in J}\left|u_{i j}(t)\right| \leq \max _{i j \in J} \frac{e^{-\lambda t} M(t)}{1-a_{i j}^{+} \eta_{i j}^{+}}=\max _{i j \in J} \frac{e^{-\lambda t} M\left(N_{0}\right)}{1-a_{i j}^{+} \eta_{i j}^{+}}<\epsilon, \quad \text { for all } t \geq t_{2} \text {. }
$$

Case (ii). If there is such a point $t_{0} \geq N_{0}$ that $M\left(t_{0}\right)=\left\|U\left(t_{0}\right)\right\|$. Then, in view of (1.5), (2.9), (2.10), (2.13), (2.16), $\left(\mathrm{T}_{1}\right)$ and $\left(\mathrm{T}_{2}\right)$, we get

$$
\begin{aligned}
0 \leq & \left.D^{-}\left(\left|U_{(i j)_{s}}(s)\right|\right)\right|_{s=t_{0}} \\
\leq & -\left(a_{(i j)_{t_{0}}}\left(t_{0}\right)-\lambda\right)\left|U_{(i j)_{t_{0}}}\left(t_{0}\right)\right|+\left(a_{(i j)_{t_{0}}}\left(t_{0}\right)-\lambda\right) \int_{t_{0}-\eta_{(i j) t_{0}}\left(t_{0}\right)}^{t_{0}} a_{(i j)_{t_{0}}}^{+} e^{\lambda s}\left|u_{(i j)_{t_{0}}}(s)\right| d s \\
& +\left|a_{(i j)_{t_{0}}}\left(t_{0}\right) e^{\lambda \eta_{(i j) t_{0}}\left(t_{0}\right)}-\left(1-\eta_{(i j)_{t_{0}}}^{\prime}(t)\right) a_{(i j)_{t_{0}}}\left(t_{0}-\eta_{(i j)_{t_{0}}}\left(t_{0}\right)\right)\right| e^{\lambda\left(t_{0}-\eta_{(i j) t_{0}}\left(t_{0}\right)\right)} \\
& \cdot\left|u_{(i j)_{t_{0}}}\left(t_{0}-\eta_{(i j) t_{0}}\left(t_{0}\right)\right)\right|+\sum_{C_{k l} \in N_{r}(i, j) t_{t_{0}}}\left|C_{(i j)_{t_{0}}}^{k l}\left(t_{0}\right)\right| e^{\lambda t_{0}} \mid f\left(x_{k l}\left(t_{0}-\tau\left(t_{0}\right)+\delta\right)\right)
\end{aligned}
$$


Liu and Shao Journal of Inequalities and Applications 2013, 2013:494

Page 11 of 22

http://www.journalofinequalitiesandapplications.com/content/2013/1/494

$$
\begin{aligned}
& -f\left(x_{k l}\left(t_{0}-\tau\left(t_{0}\right)\right)\right)|| x_{(i j) t_{0}}\left(t_{0}+\delta\right)\left|+\sum_{C_{k l} \in N_{r}(i, j) t_{t_{0}}}\right| C_{(i j)_{t_{0}}}^{k l}\left(t_{0}\right)|| f\left(x_{k l}\left(t_{0}-\tau\left(t_{0}\right)\right)\right) \mid \\
& \cdot e^{\lambda t_{0}}\left|x_{(i j)_{t_{0}}}\left(t_{0}+\delta\right)-x_{(i j)_{t_{0}}}\left(t_{0}\right)\right| \\
& +\sum_{\left.C_{k l} \in N_{q}(i, j)\right)_{t_{0}}}\left|B_{(i j)_{t_{0}}}^{k l}\left(t_{0}\right)\right| \int_{0}^{\infty}\left|K_{(i j) t_{0}}(u)\right| e^{\lambda t_{0}} \mid g\left(x_{k l}\left(t_{0}+\delta-u\right)\right) \\
& -g\left(x_{k l}\left(t_{0}-u\right)\right)|d u| x_{(i j)_{0}}\left(t_{0}+\delta\right) \mid \\
& +\sum_{C_{k l} \in N_{q}(i, j) t_{0}}\left|B_{(i j) t_{0}}^{k l}\left(t_{0}\right)\right| \int_{0}^{\infty}\left|K_{(i j)_{0}}(u)\right|\left|g\left(x_{k l}\left(t_{0}-u\right)\right)\right| d u \\
& \cdot e^{\lambda t_{0}}\left|x_{(i j) t_{0}}\left(t_{0}+\delta\right)-x_{(i j) t_{0}}\left(t_{0}\right)\right|+\left|\epsilon_{(i j) t_{0}}\left(\delta, t_{0}\right)\right| e^{\lambda t_{0}} \\
& \leq-\left(a_{(i j)_{0}}\left(t_{0}\right)-\lambda\right) M\left(t_{0}\right)+\left(a_{(i j) t_{0}}\left(t_{0}\right)-\lambda\right) a_{(i j) t_{0}}^{+} \eta_{(i j) t_{0}}^{+} \frac{M\left(t_{0}\right)}{1-a_{(i j) t_{0}}^{+} \eta_{(i j) t_{0}}^{+}} \\
& +\left|a_{(i j) t_{0}}\left(t_{0}\right) e^{\lambda \eta_{(i j) t_{0}}\left(t_{0}\right)}-\left(1-\eta_{(i j) t_{0}}^{\prime}(t)\right) a_{(i j) t_{0}}\left(t_{0}-\eta_{(i j) t_{0}}\left(t_{0}\right)\right)\right| \\
& \cdot \frac{M\left(t_{0}\right)}{1-a_{(i j)_{0}}^{+} \eta_{(i j)_{t_{0}}}^{+}}+\sum_{\left.C_{k l} \in N_{r}(i, j)\right)_{t_{0}}}\left|C_{(i j)_{t_{0}}}^{k l}\left(t_{0}\right)\right| \mu_{f} e^{\lambda \tau\left(t_{0}\right)} e^{\lambda\left(t_{0}-\tau\left(t_{0}\right)\right)}\left|u_{k l}\left(t_{0}-\tau\left(t_{0}\right)\right)\right| \\
& \frac{1}{1-a_{\left(i j t_{0}\right.}^{+} \eta_{(i j) t_{0}}^{+}}\left(\frac{L_{(i j) t_{0}}}{\delta_{(i j) t_{0}}}\right)^{+}+\sum_{C_{k l} \in N_{r}(i, j) t_{0}}\left|C_{(i j)_{0}}^{k l}\left(t_{0}\right)\right| M_{f} e^{\lambda t_{0}}\left|u_{(i j) t_{0}}\left(t_{0}\right)\right| \\
& +\sum_{C_{k l} \in N_{q}(i, j) t_{0}}\left|B_{(i j) t_{0}}^{k l}\left(t_{0}\right)\right| \int_{0}^{\infty} e^{\lambda u}\left|K_{(i j) t_{0}}(u)\right| \mu_{g} e^{\lambda\left(t_{0}-u\right)}\left|u_{k l}\left(t_{0}-u\right)\right| d u \\
& \frac{1}{1-a_{(i j) t_{0}}^{+} \eta_{(i j) t_{0}}^{+}}\left(\frac{L_{(i j) t_{0}}}{\delta_{(i j)_{0}}}\right)^{+} \\
& +\sum_{C_{k l} \in N_{q}(i, j) t_{0}}\left|B_{(i j)_{0}}^{k l}\left(t_{0}\right)\right| \int_{0}^{\infty}\left|K_{(i j)_{0}}(u)\right| d u M_{g} e^{\lambda t_{0}}\left|u_{(i j)_{t_{0}}}\left(t_{0}\right)\right|+\left|\epsilon_{(i j)_{t_{0}}}\left(\delta, t_{0}\right)\right| e^{\lambda t_{0}} \\
& \leq\left\{-\left[\left(a_{(i j) t_{0}}\left(t_{0}\right)-\lambda\right)\left(1-2 a_{(i j) t_{0}}^{+} \eta_{(i j) t_{0}}^{+}\right)-\mid a_{(i j) t_{0}}\left(t_{0}\right) e^{\lambda \eta_{(i j) t_{0}}\left(t_{0}\right)}\right.\right. \\
& \left.-\left(1-\eta_{(i j)_{0}}^{\prime}(t)\right) a_{(i j)_{t_{0}}}\left(t_{0}-\eta_{(i j)_{t_{0}}}\left(t_{0}\right)\right) \mid\right] \frac{1}{1-a_{(i j) t_{0}}^{+} \eta_{(i j) t_{0}}^{+}} \\
& +\sum_{C_{k l} \in N_{r}(i, j) t_{0}}\left|C_{(i j) t_{0}}^{k l}\left(t_{0}\right)\right| \mu_{f} e^{\lambda \tau\left(t_{0}\right)} \frac{1}{1-a_{k l}^{+} \eta_{k l}^{+}} \frac{1}{1-a_{(i j) t_{0}}^{+} \eta_{(i j) t_{0}}^{+}}\left(\frac{L_{(i j) t_{0}}}{\delta_{(i j) t_{0}}}\right)^{+} \\
& +\sum_{C_{k l} \in N_{r}(i, j) t_{0}}\left|C_{(i j)_{t_{0}}}^{k l}\left(t_{0}\right)\right| M_{f} \frac{1}{1-a_{(i j) t_{0}}^{+} \eta_{(i j)_{t_{0}}}^{+}} \\
& +\sum_{C_{k l} \in N_{q}(i, j) t_{0}}\left|B_{(i j)_{0}}^{k l}\left(t_{0}\right)\right| \int_{0}^{\infty} e^{\lambda u}\left|K_{(i j) t_{0}}(u)\right| d u \\
& \cdot \mu_{g} \frac{1}{1-a_{k l}^{+} \eta_{k l}^{+}} \frac{1}{1-a_{(i j) t_{0}}^{+} \eta_{(i j) t_{0}}^{+}}\left(\frac{L_{(i j)_{0}}}{\delta_{(i j)_{0}}}\right)^{+} \\
& \left.+\sum_{C_{k l} \in N_{q}(i, j) t_{0}}\left|B_{(i j) t_{0}}^{k l}\left(t_{0}\right)\right| \int_{0}^{\infty}\left|K_{(i j)_{0}}(u)\right| d u M_{g} \frac{1}{1-a_{(i j)_{0}}^{+} \eta_{(i j)_{0}}^{+}}\right\} M\left(t_{0}\right)
\end{aligned}
$$




$$
\begin{gathered}
+\eta \min _{i j \in J}\left\{1-a_{i j}^{+} \eta_{i j}^{+}\right\} \in e^{\lambda t_{0}} \\
<-\eta M\left(t_{0}\right)+\eta \min _{i j \in J}\left\{1-a_{i j}^{+} \eta_{i j}^{+}\right\} \epsilon e^{\lambda t_{0}},
\end{gathered}
$$

which yields that

$$
\left\|U\left(t_{0}\right)\right\|=M\left(t_{0}\right)<\min _{i j \in J}\left\{1-a_{i j}^{+} \eta_{i j}^{+}\right\} \in e^{\lambda t_{0}},
$$

and

$$
\left\|u\left(t_{0}\right)\right\| \leq \max _{i j \in J} \frac{e^{-\lambda t_{0}} M\left(t_{0}\right)}{1-a_{i j}^{+} \eta_{i j}^{+}}<\epsilon
$$

For any $t>t_{0}$, by the same approach used in the proof of (2.21) and (2.22), we have

$$
\left.\begin{array}{l}
\|U(t)\|=M(t)<\min _{i j \in J}\left\{1-a_{i j}^{+} \eta_{i j}^{+}\right\} \in e^{\lambda t} \\
\|u(t)\|<\epsilon
\end{array}\right\}, \quad \text { if } M(t)=\|U(t)\| .
$$

On the other hand, if $M(t)>\|U(t)\|$ and $t>t_{0}$, we can choose $t_{0} \leq t_{3}<t$ such that

$$
M\left(t_{3}\right)=\left\|U\left(t_{3}\right)\right\|, \quad \text { and } \quad M(s)>\|U(s)\| \quad \text { for all } s \in\left(t_{3}, t\right]
$$

which, together with (2.23), yields that

$$
M\left(t_{3}\right)=\left\|U\left(t_{3}\right)\right\|<\min _{i j \in J}\left\{1-a_{i j}^{+} \eta_{i j}^{+}\right\} \epsilon e^{\lambda t_{3}}, \quad \text { and } \quad\left\|u\left(t_{3}\right)\right\|<\epsilon .
$$

Using a similar argument as in the proof of Case (i), we can show that

$$
M(s) \equiv M\left(t_{3}\right) \text { is a constant for all } s \in\left(t_{3}, t\right]
$$

which implies that

$$
\|u(t)\| \leq \max _{i j \in J} \frac{e^{-\lambda t} M(t)}{1-a_{i j}^{+} \eta_{i j}^{+}}=\max _{i j \in J} \frac{e^{-\lambda t} M\left(t_{3}\right)}{1-a_{i j}^{+} \eta_{i j}^{+}}<\max _{i j \in J} \frac{e^{-\lambda t} \min _{i j \in J}\left\{1-a_{i j}^{+} \eta_{i j}^{+}\right\} \epsilon e^{\lambda t t_{3}}}{1-a_{i j}^{+} \eta_{i j}^{+}}<\epsilon .
$$

In summary, there must exist $N>\max \left\{t_{0}, N_{0}, t_{2}\right\}$ such that $\|u(t)\| \leq \epsilon$ holds for all $t>N$. The proof of Lemma 2.2 is now complete.

\section{Main results}

In this section, we establish some results for the existence, uniqueness and exponential stability of the almost periodic solution of (1.2).

Theorem 3.1 Suppose that $\left(\mathrm{T}_{1}\right)$ and $\left(\mathrm{T}_{2}\right)$ are satisfied. Then system (1.2) has exactly one almost periodic solution $Z^{*}(t)$. Moreover, $Z^{*}(t)$ is globally exponentially stable.

Proof Let $v(t)=\left\{v_{i j}(t)\right\}$ be a solution of system (1.2) with initial function $\varphi_{i j}^{v}(\cdot)$ satisfying $(2.1)$, and $\left(\varphi_{i j}^{v}(\cdot)\right)^{\prime}$ is bounded continuous on $(-\infty, 0]$. 
Set

$$
\begin{aligned}
\epsilon_{i j, k}(t)= & -a_{i j}(t)\left[x_{i j}\left(t+t_{k}-\eta_{i j}\left(t+t_{k}\right)\right)-x_{i j}\left(t-\eta_{i j}(t)+t_{k}\right)\right] \\
& -\left[a_{i j}\left(t+t_{k}\right)-a_{i j}(t)\right] x_{i j}\left(t+t_{k}-\eta_{i j}\left(t+t_{k}\right)\right) \\
& -\sum_{C_{k l} \in N_{r}(i, j)}\left[C_{i j}^{k l}\left(t+t_{k}\right)-C_{i j}^{k l}(t)\right] f\left(x_{k l}\left(t-\tau\left(t+t_{k}\right)+t_{k}\right)\right) x_{i j}\left(t+t_{k}\right) \\
& -\sum_{C_{k l} \in N_{r}(i, j)} C_{i j}^{k l}(t)\left[f\left(x_{k l}\left(t-\tau\left(t+t_{k}\right)+t_{k}\right)\right)-f\left(x_{k l}\left(t-\tau(t)+t_{k}\right)\right)\right] x_{i j}\left(t+t_{k}\right) \\
& -\sum_{C_{k l} \in N_{q}(i, j)}\left[B_{i j}^{k l}\left(t+t_{k}\right)-B_{i j}^{k l}(t)\right] \int_{0}^{\infty} K_{i j}(u) g\left(x_{k l}\left(t+t_{k}-u\right)\right) d u x_{i j}\left(t+t_{k}\right) \\
& +\left[L_{i j}\left(t+t_{k}\right)-L_{i j}(t)\right], \quad i j \in J,
\end{aligned}
$$

where $\left\{t_{k}\right\}$ is any sequence of real numbers. By Lemma 2.1, the solution $v(t)$ is bounded and

$$
\left|v_{i j}(t)\right| \leq \frac{1}{1-a_{i j}^{+} \eta_{i j}^{+}}\left(\frac{L_{i j}}{\delta_{i j}}\right)^{+} \text {for all } t \in R, i j \in J
$$

which implies that the right side of (1.2) is also bounded, and $v^{\prime}(t)$ is a bounded function on $R$. Thus, $v(t)$ is uniformly continuous on $R$. Then, from the almost periodicity of $a_{i j}, \eta_{i j}$, $\tau, C_{i j}^{k l}$ and $B_{i j}^{k l}$, we can select a sequence $\left\{t_{k}\right\} \rightarrow+\infty$ such that

$$
\left.\begin{array}{ll}
\left|a_{i j}\left(t+t_{k}\right)-a_{i j}(t)\right| \leq \frac{1}{k}, & \left|C_{i j}^{k l}\left(t+t_{k}\right)-C_{i j}^{k l}(t)\right| \leq \frac{1}{k}, \quad\left|B_{i j}^{k l}\left(t+t_{k}\right)-B_{i j}^{k l}(t)\right| \leq \frac{1}{k} \\
\left|\eta_{i j}\left(t+t_{k}\right)-\eta_{i j}(t)\right| \leq \frac{1}{k}, & \left|\tau\left(t+t_{k}\right)-\tau(t)\right| \leq \frac{1}{k}, \quad\left|\epsilon_{i j, k}(t)\right| \leq \frac{1}{k}
\end{array}\right\}
$$

for all $i j, k l \in J, t \in R$.

Since $\left\{v\left(t+t_{k}\right)\right\}_{k=1}^{+\infty}$ is uniformly bounded and equiuniformly continuous, by ArzalaAscoli lemma and diagonal selection principle, we can choose a subsequence $\left\{t_{k_{j}}\right\}$ of $\left\{t_{k}\right\}$, such that $v\left(t+t_{k_{j}}\right)$ (for convenience, we still denote by $v\left(t+t_{k}\right)$ ) uniformly converges to a continuous function $Z^{*}(t)=\left\{x_{i j}^{*}(t)\right\}$ on any compact set of $R$, and

$$
\left|x_{i j}^{*}(t)\right| \leq \frac{1}{1-a_{i j}^{+} \eta_{i j}^{+}}\left(\frac{L_{i j}}{\delta_{i j}}\right)^{+} \text {for all } t \in R, i j \in J
$$

Now, we prove that $Z^{*}(t)$ is a solution of (1.2). In fact, for any $t>0$ and $\Delta t \in R$, from (3.3), we have

$$
\begin{aligned}
x_{i j}^{*}(t+\Delta t)-x_{i j}^{*}(t) \\
=\lim _{k \rightarrow+\infty}\left[v_{i j}\left(t+\Delta t+t_{k}\right)-v_{i j}\left(t+t_{k}\right)\right] \\
=\lim _{k \rightarrow+\infty} \int_{t}^{t+\Delta t}\left\{-a_{i j}\left(\mu+t_{k}\right) v_{i j}\left(\mu+t_{k}-\eta_{i j}\left(\mu+t_{k}\right)\right)\right. \\
\quad-\sum_{C_{k l} \in N_{r}(i, j)} C_{i j}^{k l}\left(\mu+t_{k}\right) f\left(v_{k l}\left(\mu+t_{k}-\tau\left(\mu+t_{k}\right)\right)\right) v_{i j}\left(\mu+t_{k}\right)
\end{aligned}
$$




$$
\begin{aligned}
& \left.-\sum_{C_{k l} \in N_{q}(i, j)} B_{i j}^{k l}\left(\mu+t_{k}\right) \int_{0}^{\infty} K_{i j}(u) g\left(v_{k l}\left(\mu+t_{k}-u\right)\right) d u v_{i j}\left(\mu+t_{k}\right)+L_{i j}\left(\mu+t_{k}\right)\right\} d \mu \\
= & \lim _{k \rightarrow+\infty} \int_{t}^{t+\Delta t}\left\{-a_{i j}(\mu) v_{i j}\left(\mu-\eta_{i j}(\mu)+t_{k}\right)\right. \\
& -\sum_{C_{k l} \in N_{r}(i, j)} C_{i j}^{k l}(\mu) f\left(v_{k l}\left(\mu-\tau(\mu)+t_{k}\right)\right) v_{i j}\left(\mu+t_{k}\right) \\
& \left.-\sum_{C_{k l} \in N_{q}(i, j)} B_{i j}^{k l}(\mu) \int_{0}^{\infty} K_{i j}(u) g\left(v_{k l}\left(\mu+t_{k}-u\right)\right) d u v_{i j}\left(\mu+t_{k}\right)+L_{i j}(\mu)+\epsilon_{i j, k}(\mu)\right\} d \mu \\
= & \int_{t}^{t+\Delta t}\left\{-a_{i j}(\mu) x_{i j}^{*}\left(\mu-\eta_{i j}(\mu)\right)-\sum_{C_{k l} \in N_{r}(i, j)} C_{i j}^{k l}(\mu) f\left(x_{k l}^{*}(\mu-\tau(\mu))\right) x_{i j}^{*}(\mu)\right. \\
& \left.-\sum_{C_{k l} \in N_{q}(i, j)} B_{i j}^{k l}(\mu) \int_{0}^{\infty} K_{i j}(u) g\left(x_{k l}^{*}(\mu-u)\right) d u x_{i j}^{*}(\mu)+L_{i j}(\mu)\right\} d \mu \\
& +\lim _{k \rightarrow+\infty} \int_{t}^{t+\Delta t} \epsilon_{i j, k}(\mu) d \mu \\
= & \int_{t}^{t+\Delta t}\left\{-a_{i j}(\mu) x_{i j}^{*}\left(\mu-\eta_{i j}(\mu)\right)-\sum_{C_{k l} \in N_{r}(i, j)} C_{i j}^{k l}(\mu) f\left(x_{k l}^{*}(\mu-\tau(\mu))\right) x_{i j}^{*}(\mu)\right. \\
& \left.-\sum_{C_{k l} \in N_{q}(i, j)} B_{i j}^{k l}(\mu) \int_{0}^{\infty} K_{i j}(u) g\left(x_{k l}^{*}(\mu-u)\right) d u x_{i j}^{*}(\mu)+L_{i j}(\mu)\right\} d \mu,
\end{aligned}
$$

which implies that

$$
\begin{aligned}
\frac{d}{d t}\left\{x_{i j}^{*}(t)\right\}= & -a_{i j}(t) x_{i j}^{*}\left(t-\eta_{i j}(t)\right)-\sum_{C_{k l} \in N_{r}(i, j)} C_{i j}^{k l}(t) f\left(x_{k l}^{*}(t-\tau(t))\right) x_{i j}^{*}(t) \\
& -\sum_{C_{k l} \in N_{q}(i, j)} B_{i j}^{k l}(t) \int_{0}^{\infty} K_{i j}(u) g\left(x_{k l}^{*}(t-u)\right) d u x_{i j}^{*}(t)+L_{i j}(t), \quad i j \in J .
\end{aligned}
$$

Therefore, $Z^{*}(t)$ is a solution of (1.2).

Secondly, we prove that $Z^{*}(t)$ is a almost periodic solution of (1.2). From Lemma 2.2, for any $\varepsilon>0$, there exists $l=l(\varepsilon)>0$, such that every interval $[\alpha, \alpha+l]$ contains at least one number $\delta$ for which there exists $N>0$ satisfying

$$
\left|v_{i j}(t+\delta)-v_{i j}(t)\right| \leq \varepsilon \quad \text { for all } t>N, i j \in J
$$

Then, for any fixed $s \in R$, we can find a sufficient large positive integer $N_{1}>N$ such that for any $k>N_{1}$

$$
s+t_{k}>N, \quad\left|v_{i j}\left(s+t_{k}+\delta\right)-v_{i j}\left(s+t_{k}\right)\right| \leq \varepsilon, \quad i j \in J .
$$

Let $k \rightarrow+\infty$, we obtain

$$
\left|x_{i j}^{*}(s+\delta)-x_{i j}^{*}(s)\right| \leq \varepsilon, \quad i j \in J,
$$

which implies that $Z^{*}(t)$ is an almost periodic solution of (1.2). 
Finally, we prove that $Z^{*}(t)$ is globally exponentially stable.

Let $Z^{*}(t)=\left\{x_{i j}^{*}(t)\right\}$ be the positive almost periodic solution of system (1.2) with initial value $\varphi^{*}=\left\{\varphi_{i j}^{*}(t)\right\}$, and let $Z(t)=\left\{x_{i j}(t)\right\}$ be an arbitrary solution of system (1.2) with initial value $\varphi=\left\{\varphi_{i j}(t)\right\}$, set $y(t)=\left\{y_{i j}(t)\right\}=\left\{x_{i j}(t)-x_{i j}^{*}(t)\right\}=Z(t)-Z^{*}(t)$. Then

$$
\begin{aligned}
y_{i j}^{\prime}(t)= & -a_{i j}(t) y_{i j}\left(t-\eta_{i j}(t)\right)-\sum_{C_{k l} \in N_{r}(i, j)} C_{i j}^{k l}(t)\left[f\left(x_{k l}(t-\tau(t))\right) x_{i j}(t)\right. \\
& \left.-f\left(x_{k l}^{*}(t-\tau(t))\right) x_{i j}^{*}(t)\right]-\sum_{C_{k l} \in N_{q}(i, j)} B_{i j}^{k l}(t)\left[\int_{0}^{\infty} K_{i j}(u) g\left(x_{k l}(t-u)\right) d u x_{i j}(t)\right. \\
& \left.-\int_{0}^{\infty} K_{i j}(u) g\left(x_{k l}^{*}(t-u)\right) d u x_{i j}^{*}(t)\right],
\end{aligned}
$$

which yields

$$
\begin{aligned}
& \frac{d}{d t}(\left.e^{\lambda t} y_{i j}(t)-\int_{t-\eta_{i j}(t)}^{t} a_{i j}(s) e^{\lambda s} y_{i j}(s) d s\right) \\
&= \lambda e^{\lambda t} y_{i j}(t)+e^{\lambda t} y_{i j}^{\prime}(t) \\
&-\left[a_{i j}(t) e^{\lambda t} y_{i j}(t)-\left(1-\eta_{i j}^{\prime}(t)\right) a_{i j}\left(t-\eta_{i j}(t)\right) e^{\lambda\left(t-\eta_{i j}(t)\right)} y_{i j}\left(t-\eta_{i j}(t)\right)\right] \\
&= \lambda e^{\lambda t} y_{i j}(t)-\left[a_{i j}(t) e^{\lambda t} y_{i j}(t)-\left(1-\eta_{i j}^{\prime}(t)\right) a_{i j}\left(t-\eta_{i j}(t)\right) e^{\lambda\left(t-\eta_{i j}(t)\right)} y_{i j}\left(t-\eta_{i j}(t)\right)\right] \\
&+e^{\lambda t}\left\{-a_{i j}(t) y_{i j}\left(t-\eta_{i j}(t)\right)-\sum_{C_{k l} \in N_{r}(i, j)} C_{i j}^{k l}(t)\left[f\left(x_{k l}(t-\tau(t))\right) x_{i j}(t)\right.\right. \\
&\left.-f\left(x_{k l}^{*}(t-\tau(t))\right) x_{i j}^{*}(t)\right]-\sum_{C_{k l} \in N_{q}(i, j)} B_{i j}^{k l}(t)\left[\int_{0}^{\infty} K_{i j}(u) g\left(x_{k l}(t-u)\right) d u x_{i j}(t)\right. \\
&\left.\left.-\int_{0}^{\infty} K_{i j}(u) g\left(x_{k l}^{*}(t-u)\right) d u x_{i j}^{*}(t)\right]\right\} \\
&=-\left(a_{i j}(t)-\lambda\right) e^{\lambda t} y_{i j}(t) \\
&-\left[a_{i j}(t)-\left(1-\eta_{i j}^{\prime}(t)\right) a_{i j}\left(t-\eta_{i j}(t)\right) e^{-\lambda \eta_{i j}(t)}\right] e^{\lambda t} y_{i j}\left(t-\eta_{i j}(t)\right) \\
&+e^{\lambda t}\left\{-\sum_{C_{k l} \in N_{r}(i, j)} C_{i j}^{k l}(t)\left[f\left(x_{k l}(t-\tau(t))\right) x_{i j}(t)-f\left(x_{k l}^{*}(t-\tau(t))\right) x_{i j}^{*}(t)\right]\right. \\
&-\left[a_{i j}(t)-\left(1-\eta_{i j}^{\prime}(t)\right) a_{i j}\left(t-\eta_{i j}(t)\right) e^{-\lambda \eta_{i j}(t)}\right] e^{\lambda t} y_{i j}\left(t-\eta_{i j}(t)\right) \\
&-\sum_{k+N_{q}(i, j)} B_{i j}^{k l}(t)\left[\int_{0}^{\infty} K_{i j}(u) g\left(x_{k l}(t-u)\right) d u x_{i j}(t)\right. \\
&\left.\left.-\int_{0}^{\infty} K_{i j}(u) g\left(x_{k l}^{*}(t-u)\right) d u x_{i j}^{*}(t)\right]\right\} \\
&=-\left(a_{i j}(t)-\lambda\right)\left(e^{\lambda t} y_{i j}(t)-\int_{t-\eta_{i j}(t)}^{t} a_{i j}(s) e^{\lambda s} y_{i j}(s) d s\right) \\
& a_{i j}(s) e^{\lambda s} y_{i j}(s) d s \\
& \\
&
\end{aligned}
$$




$$
\begin{aligned}
& +e^{\lambda t}\left\{-\sum_{C_{k l} \in N_{r}(i, j)} C_{i j}^{k l}(t)\left[f\left(x_{k l}(t-\tau(t))\right)-f\left(x_{k l}^{*}(t-\tau(t))\right)\right] x_{i j}(t)\right. \\
& -\sum_{C_{k l} \in N_{r}(i, j)} C_{i j}^{k l}(t) f\left(x_{k l}^{*}(t-\tau(t))\right)\left[x_{i j}(t)-x_{i j}^{*}(t)\right] \\
& -\sum_{C_{k l} \in N_{q}(i, j)} B_{i j}^{k l}(t) \int_{0}^{\infty} K_{i j}(u)\left[g\left(x_{k l}(t-u)\right)-g\left(x_{k l}^{*}(t-u)\right)\right] d u x_{i j}(t) \\
& \left.-\sum_{C_{k l} \in N_{q}(i, j)} B_{i j}^{k l}(t) \int_{0}^{\infty} K_{i j}(u) g\left(x_{k l}^{*}(t-u)\right) d u\left[x_{i j}(t)-x_{i j}^{*}(t)\right]\right\}
\end{aligned}
$$

where $i j \in J$.

Let

$$
Y_{i j}(t)=\left|e^{\lambda t} y_{i j}(t)-\int_{t-\eta_{i j}(t)}^{t} a_{i j}(s) e^{\lambda s} y_{i j}(s) d s\right|, \quad i j \in J
$$

We define a positive constant $M$ as follows:

$$
M=\max _{i j \in J}\left\{\sup _{s \in(-\infty, 0]} Y_{i j}(s)\right\}
$$

Let $K$ be a positive number such that

$$
Y_{i j}(t) \leq M<M+1=K \quad \text { for all } t \in(-\infty, 0], i j \in J .
$$

We claim that

$$
Y_{i j}(t)<K, \quad \text { for all } t>0, i=1,2, \ldots, n \text {. }
$$

Otherwise, there must exist $i j \in J$ and $\theta>0$ such that

$$
Y_{i j}(\theta)=K, \quad Y_{\tilde{i} j}(t)<K, \quad \text { for all } t \in(-\infty, \theta), \tilde{i j} \in J
$$

It follows that

$$
\begin{aligned}
e^{\lambda t}\left|y_{\tilde{i} j}(t)\right| \leq & \left|e^{\lambda t} y_{\tilde{i} j}(t)-\int_{t-\eta_{\tilde{i} j}(t)}^{t} a_{\tilde{i} j}(s) e^{\lambda s} y_{\tilde{i} j}(s) d s\right| \\
& +\left|\int_{t-\eta_{\tilde{i j}}(t)}^{t} a_{\tilde{i} j}(s) e^{\lambda s} y_{\tilde{i} j}(s) d s\right| \\
\leq & K+a_{\tilde{i j}}^{+} \eta_{\tilde{i} j}^{+} \sup _{s \in(-\infty, \theta]} e^{\lambda s}\left|y_{\tilde{i} j}(s)\right|, \quad \text { for all } t \in(-\infty, \theta], \tilde{i j} \in J .
\end{aligned}
$$

Consequently, in view of (3.14) and the fact $a_{i j}^{+} \eta_{\tilde{i} j}^{+}<1(\tilde{i j} \in J)$, we have

$$
e^{\lambda t}\left|y_{\tilde{i} j}(t)\right| \leq \sup _{s \in(-\infty, \theta]} e^{\lambda s}\left|y_{\tilde{i} j}(s)\right| \leq \frac{K}{1-a_{\tilde{i} j}^{+} \eta_{\tilde{i} j}^{+}} \quad \text { for all } t \in(-\infty, \theta], \tilde{i j} \in J
$$


Li and Shoo Journal of Inequalities and Applications 2013, 2013:494

Page 17 of 22

http://www.journalofinequalitiesandapplications.com/content/2013/1/494

Calculating the upper left derivative of $Y_{i j}(t)$, together with (2.3), (3.10), (3.13), (3.15), $\left(\mathrm{T}_{1}\right)$ and $\left(\mathrm{T}_{2}\right)$, we obtain

$$
\begin{aligned}
& 0 \leq D^{-} Y_{i j}(\theta) \\
& \leq-\left(a_{i j}(\theta)-\lambda\right) Y_{i j}(\theta)+\mid-\left(a_{i j}(\theta)-\lambda\right) \int_{\theta-\eta_{i j}(\theta)}^{\theta} a_{i j}(s) e^{\lambda s} y_{i j}(s) d s \\
& -\left[a_{i j}(\theta)-\left(1-\eta_{i j}^{\prime}(\theta)\right) a_{i j}\left(\theta-\eta_{i j}(\theta)\right) e^{-\lambda \eta_{i j}(\theta)}\right] e^{\lambda \theta} y_{i j}\left(\theta-\eta_{i j}(\theta)\right) \\
& +e^{\lambda \theta}\left\{-\sum_{C_{k l} \in N_{r}(i, j)} C_{i j}^{k l}(\theta)\left[f\left(x_{k l}(\theta-\tau(\theta))\right)-f\left(x_{k l}^{*}(\theta-\tau(\theta))\right)\right] x_{i j}(\theta)\right. \\
& -\sum_{C_{k l} \in N_{r}(i, j)} C_{i j}^{k l}(\theta) f\left(x_{k l}^{*}(\theta-\tau(\theta))\right)\left[x_{i j}(\theta)-x_{i j}^{*}(\theta)\right] \\
& -\sum_{C_{k l} \in N_{q}(i, j)} B_{i j}^{k l}(\theta) \int_{0}^{\infty} K_{i j}(u)\left[g\left(x_{k l}(\theta-u)\right)-g\left(x_{k l}^{*}(\theta-u)\right)\right] d u x_{i j}(\theta) \\
& \left.-\sum_{C_{k l} \in N_{q}(i, j)} B_{i j}^{k l}(\theta) \int_{0}^{\infty} K_{i j}(u) g\left(x_{k l}^{*}(\theta-u)\right) d u\left[x_{i j}(\theta)-x_{i j}^{*}(\theta)\right]\right\} \mid \\
& \leq-\left(a_{i j}(\theta)-\lambda\right) Y_{i j}(\theta)+\left(a_{i j}(\theta)-\lambda\right) \frac{K}{1-a_{i j}^{+} \eta_{i j}^{+}} a_{i j}^{+} \eta_{i j}^{+} \\
& +\left|a_{i j}(\theta) e^{\lambda \eta_{i j}(\theta)}-\left(1-\eta_{i j}^{\prime}(\theta)\right) a_{i j}\left(\theta-\eta_{i j}(\theta)\right)\right| e^{\lambda\left(\theta-\eta_{i j}(\theta)\right)}\left|y_{i j}\left(\theta-\eta_{i j}(\theta)\right)\right| \\
& +\sum_{C_{k l} \in N_{r}(i, j)}\left|C_{i j}^{k l}(\theta)\right| \mu_{f} e^{\lambda \tau(\theta)} e^{\lambda(\theta-\tau(\theta))}\left|y_{k l}(\theta-\tau(\theta))\right|\left|x_{i j}(\theta)\right| \\
& +\sum_{C_{k l} \in N_{r}(i, j)}\left|C_{i j}^{k l}(\theta)\right| M_{f} e^{\lambda \theta}\left|y_{i j}(\theta)\right| \\
& +\sum_{C_{k l} \in N_{q}(i, j)}\left|B_{i j}^{k l}(\theta)\right| \int_{0}^{\infty} e^{\lambda u}\left|K_{i j}(u)\right| \mu_{g} e^{\lambda(\theta-u)}\left|y_{k l}(\theta-u)\right| d u\left|x_{i j}(\theta)\right| \\
& +\sum_{C_{k l} \in N_{q}(i, j)}\left|B_{i j}^{k l}(\theta)\right| \int_{0}^{\infty}\left|K_{i j}(u)\right| d u M_{g} e^{\lambda \theta}\left|y_{i j}(\theta)\right| \\
& \leq\left\{-\left[\left(a_{i j}(\theta)-\lambda\right)\left(1-2 a_{i j}^{+} \eta_{i j}^{+}\right)\right.\right. \\
& \left.-\left|a_{i j}(\theta) e^{\lambda \eta_{i j}(\theta)}-\left(1-\eta_{i j}^{\prime}(\theta)\right) a_{i j}\left(\theta-\eta_{i j}(\theta)\right)\right|\right] \frac{1}{1-a_{i j}^{+} \eta_{i j}^{+}} \\
& +\sum_{C_{k l} \in N_{r}(i, j)}\left|C_{i j}^{k l}(\theta)\right| \mu_{f} e^{\lambda \tau(\theta)} \frac{1}{1-a_{k l}^{+} \eta_{k l}^{+}} \frac{1}{1-a_{i j}^{+} \eta_{i j}^{+}}\left(\frac{L_{i j}}{\delta_{i j}}\right)^{+} \\
& +\sum_{C_{k l} \in N_{r}(i, j)}\left|C_{i j}^{k l}(\theta)\right| M_{f} \frac{1}{1-a_{i j}^{+} \eta_{i j}^{+}} \\
& +\sum_{C_{k l} \in N_{q}(i, j)}\left|B_{i j}^{k l}(\theta)\right| \int_{0}^{\infty} e^{\lambda u}\left|K_{i j}(u)\right| d u \mu_{g} \frac{1}{1-a_{k l}^{+} \eta_{k l}^{+}} \frac{1}{1-a_{i j}^{+} \eta_{i j}^{+}}\left(\frac{L_{i j}}{\delta_{i j}}\right)^{+} \\
& \left.+\sum_{C_{k l} \in N_{q}(i, j)}\left|B_{i j}^{k l}(\theta)\right| \int_{0}^{\infty}\left|K_{i j}(u)\right| d u M_{g} \frac{1}{1-a_{i j}^{+} \eta_{i j}^{+}}\right\} K
\end{aligned}
$$




$$
\begin{aligned}
& <-\eta K \\
& <0,
\end{aligned}
$$

which is a contradiction, and it implies that (3.12) holds.

Consequently, using a similar argument as in (3.14)-(3.15), we know that

$$
\left|y_{i j}(t)\right| e^{\lambda t}<\frac{K}{1-a_{i j}^{+} \eta_{i j}^{+}}, \quad \text { for all } t \in R, i j \in J
$$

That is,

$$
\left|x_{i j}(t)-x_{i j}^{*}(t)\right| \leq \frac{K}{1-a_{i j}^{+} \eta_{i j}^{+}} e^{-\lambda t}, \quad \text { for all } t>0 \text {, and } i j \in J
$$

This completes the proof.

\section{An example}

In this section, we give an example with numerical simulations to demonstrate the results obtained in previous sections.

Example 4.1 Consider the following SICNNs with time-varying delays in the leakage terms:

$$
\begin{aligned}
& \frac{d x_{i j}}{d t}=-a_{i j}(t) x_{i j}\left(t-\eta_{i j}(t)\right)-\sum_{C_{k l} \in N_{r}(i, j)} C_{i j}^{k l} f\left(x_{k l}\left(t-\sin ^{2} t\right)\right) x_{i j}(t) \\
& -\sum_{C_{k l} \in N_{q}(i, j)} B_{i j}^{k l} \int_{0}^{\infty} K_{i j}(u) g\left(x_{k l}(t-u)\right) d u x_{i j}+L_{i j}(t), \quad i, j=1,2,3, \\
& {\left[\begin{array}{lll}
a_{11} & a_{12} & a_{13} \\
a_{21} & a_{22} & a_{23} \\
a_{31} & a_{32} & a_{33}
\end{array}\right]=\left[\begin{array}{lll}
1 & 1 & 3 \\
3 & 1 & 3 \\
3 & 1 & 3
\end{array}\right],} \\
& {\left[\begin{array}{lll}
B_{11} & B_{12} & B_{13} \\
B_{21} & B_{22} & B_{23} \\
B_{31} & B_{32} & B_{33}
\end{array}\right]=\left[\begin{array}{lll}
C_{11} & C_{12} & C_{13} \\
C_{21} & C_{22} & C_{23} \\
C_{31} & C_{32} & C_{33}
\end{array}\right]=\left[\begin{array}{ccc}
0.1 & 0.2 & 0.1 \\
0.2 & 0 & 0.2 \\
0.1 & 0.2 & 0.1
\end{array}\right],} \\
& {\left[\begin{array}{lll}
\eta_{11} & \eta_{12} & \eta_{13} \\
\eta_{21} & \eta_{22} & \eta_{23} \\
\eta_{31} & \eta_{32} & \eta_{33}
\end{array}\right]=0.01\left[\begin{array}{ccc}
\sin ^{2} \sqrt{3} t & \cos ^{2} \sqrt{3} t & \sin ^{2} 2 t \\
\cos ^{2} \sqrt{5} t & \sin ^{2} \sqrt{5} t & \cos ^{2} 2 t \\
\sin ^{2} 2 t & \cos ^{2} 3 t & \sin ^{2} \sqrt{2} t
\end{array}\right] \text {, }} \\
& {\left[\begin{array}{lll}
L_{11} & L_{12} & L_{13} \\
L_{21} & L_{22} & L_{23} \\
L_{31} & L_{32} & L_{33}
\end{array}\right]} \\
& =\left[\begin{array}{ccc}
0.7+0.24 \sin ^{2} \sqrt{2} t & 0.41+0.5 \cos ^{2} t & 0.74+0.2 \sin ^{2} t \\
0.61+0.2 \cos ^{2} t & 0.67+0.2 \sin ^{2} t & 0.75+0.2 \sin ^{2} t \\
0.59+0.4 \cos ^{4} t & 0.5+0.41 \sin ^{2} t & 0.76+0.2 \cos ^{2} t
\end{array}\right] .
\end{aligned}
$$


Set

$$
\lambda=0.001, \quad r=q=1, \quad K_{i j}(u)=|\sin u| e^{-u}, \quad i=1,2,3, j=1,2,3,
$$

and

$$
f(x)=g(x)=\frac{1}{40}(|x-1|-|x+1|)
$$

clearly,

$$
\begin{aligned}
& M_{f}=M_{g}=0.05, \quad \mu_{f}=\mu_{g}=0.05, \\
& \sum_{C_{k l} \in N_{1}(1,1)} C_{11}^{k l}=\sum_{C_{k l} \in N_{1}(1,1)} B_{11}^{k l}=0.5, \quad \sum_{C_{k l} \in N_{1}(1,2)} C_{12}^{k l}=\sum_{C_{k l} \in N_{1}(1,2)} B_{12}^{k l}=0.8, \\
& \sum_{C_{k l} \in N_{1}(1,3)} C_{13}^{k l}=\sum_{C_{k l} \in N_{1}(1,3)} B_{13}^{k l}=0.5, \quad \sum_{C_{k l} \in N_{1}(2,1)} C_{21}^{k l}=\sum_{C_{k l} \in N_{1}(2,1)} B_{21}^{k l}=0.8, \\
& \sum_{C_{k l} \in N_{1}(2,2)} C_{22}^{k l}=\sum_{C_{k l} \in N_{1}(2,2)} B_{22}^{k l}=1.2, \quad \sum_{C_{k l} \in N_{1}(2,3)} C_{23}^{k l}=\sum_{C_{k l} \in N_{1}(2,3)} B_{23}^{k l}=0.8, \\
& \sum_{C_{k l} \in N_{1}(3,1)} C_{31}^{k l}=\sum_{C_{k l} \in N_{1}(3,1)} B_{31}^{k l}=0.5, \quad \sum_{C_{k l} \in N_{1}(3,2)} C_{32}^{k l}=\sum_{C_{k l} \in N_{1}(3,2)} B_{32}^{k l}=0.8, \\
& C_{33}^{k l}=\sum_{C_{k l} \in N_{1}(3,3)} B_{33}^{k l}=0.5, \\
& \eta_{i j}^{+} \leq 0.01, \\
& a_{i j}^{+} \leq 3,
\end{aligned}
$$

where $i j \in J=\{11,12,13,21,22,23,31,32,33\}$. Then,

$$
\begin{aligned}
& \min _{i j \in J} \delta_{i j}(t)=\min _{i j \in J}\left\{\left\{a_{i j}(t)\left(1-2 a_{i j}^{+} \eta_{i j}^{+}\right)-\left|a_{i j}(t)-\left(1-\eta_{i j}^{\prime}(t)\right) a_{i j}\left(t-\eta_{i j}(t)\right)\right|\right.\right. \\
& \left.\left.-\sum_{C_{k l} \in N_{r}(i, j)}\left|C_{i j}^{k l}(t)\right| M_{f}-\sum_{C_{k l} \in N_{q}(i, j)}\left|B_{i j}^{k l}(t)\right| \int_{0}^{\infty}\left|K_{i j}(u)\right| d u M_{g}\right\} \frac{1}{1-a_{i j}^{+} \eta_{i j}^{+}}\right\} \\
& >0.5>0, \quad \text { for all } t \geq 0 \text {, } \\
& \max _{i j \in J}\left\{-\left[\left(a_{i j}(t)-\lambda\right)\left(1-2 a_{i j}^{+} \eta_{i j}^{+}\right)-\left|a_{i j}(t) e^{\lambda \eta_{i j}(t)}-\left(1-\eta_{i j}^{\prime}(t)\right) a_{i j}\left(t-\eta_{i j}(t)\right)\right|\right] \frac{1}{1-a_{i j}^{+} \eta_{i j}^{+}}\right. \\
& +\sum_{C_{k l} \in N_{r}(i, j)}\left|C_{i j}^{k l}(t)\right| \mu_{f} e^{\lambda \tau(t)} \frac{1}{1-a_{k l}^{+} \eta_{k l}^{+}} \frac{1}{1-a_{i j}^{+} \eta_{i j}^{+}}\left(\frac{L_{i j}}{\delta_{i j}}\right)^{+} \\
& +\sum_{C_{k l} \in N_{r}(i, j)}\left|C_{i j}^{k l}(t)\right| M_{f} \frac{1}{1-a_{i j}^{+} \eta_{i j}^{+}} \\
& +\sum_{C_{k l} \in N_{q}(i, j)}\left|B_{i j}^{k l}(t)\right| \int_{0}^{\infty} e^{\lambda u}\left|K_{i j}(u)\right| d u \mu_{g} \frac{1}{1-a_{k l}^{+} \eta_{k l}^{+}} \frac{1}{1-a_{i j}^{+} \eta_{i j}^{+}}\left(\frac{L_{i j}}{\delta_{i j}}\right)^{+} \\
& \left.+\sum_{C_{k l} \in N_{q}(i, j)}\left|B_{i j}^{k l}(t)\right| \int_{0}^{\infty}\left|K_{i j}(u)\right| d u M_{g} \frac{1}{1-a_{i j}^{+} \eta_{i j}^{+}}\right\} \\
& <-(1-\lambda) \times(1-2 \times 1 \times 0.01)+\left(1 \times e^{0.01 \lambda}-1+0.01\right) \frac{1}{1-0.01}
\end{aligned}
$$




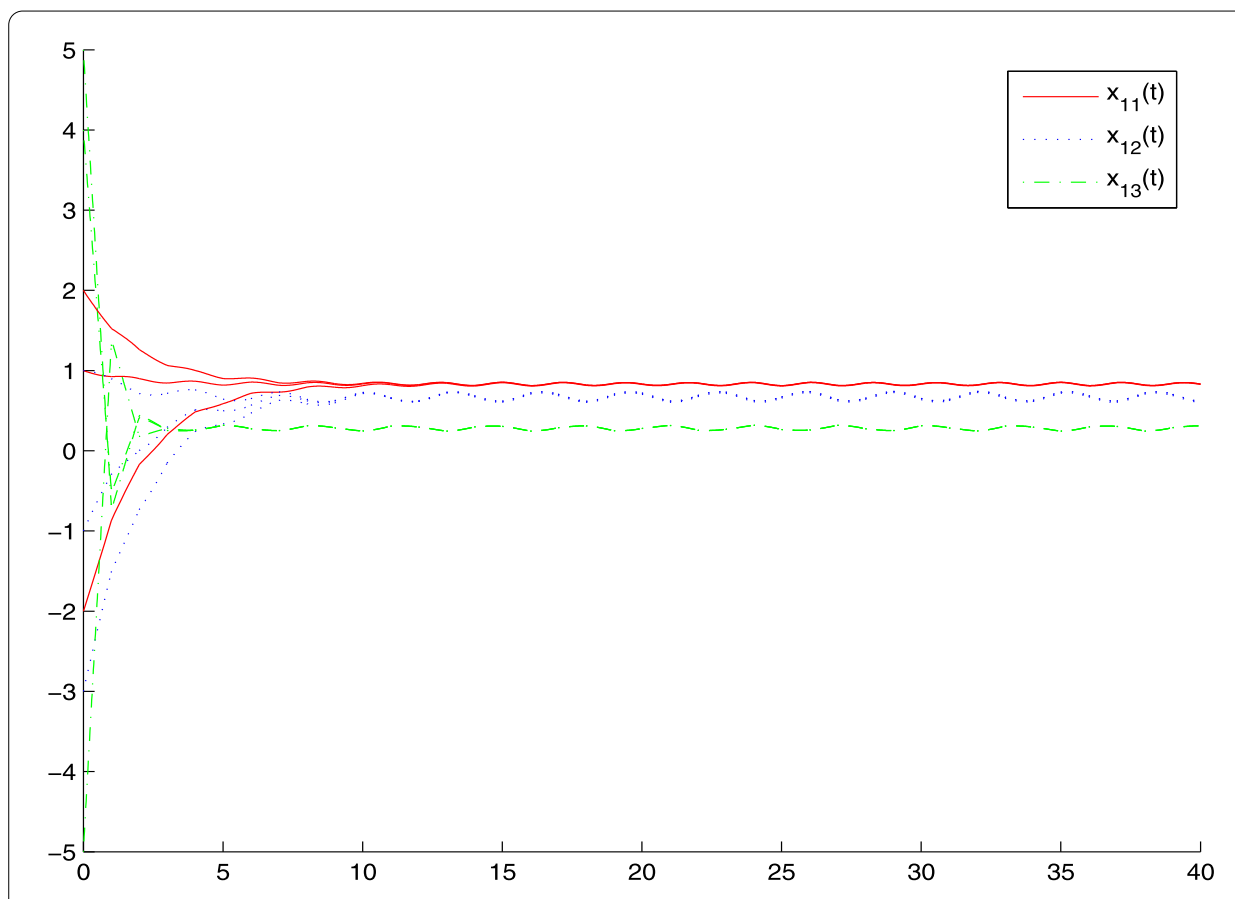

Figure 1 Numerical solutions of system (4.1) for different initial values.

$$
\begin{aligned}
& +1.2 \times 0.05 \times e^{\lambda \times 1} \frac{1}{1-0.03} \frac{1}{1-0.03} \times 2+1.2 \times 0.05 \times \frac{1}{1-0.03} \\
& +1.2 \times \frac{e^{\lambda}}{1-\lambda} \times 0.05 \frac{1}{1-0.03} \frac{1}{1-0.03}+1.2 \times 1 \times 0.05 \times \frac{1}{1-0.03} \\
& \approx-0.6538<-0.5, \quad \text { for all } t \geq 0 .
\end{aligned}
$$

It follows that system (4.1) satisfies all the conditions in Theorem 3.1. Hence, system (4.1) has exactly one positive almost periodic solution. Moreover, the almost periodic solution is globally exponentially stable. The fact is verified by the numerical simulation in Figures 1-3 and their three different initial values, which are $\varphi_{11} \equiv 1, \varphi_{12} \equiv-3, \varphi_{13} \equiv 4$, $\varphi_{21} \equiv 2, \varphi_{22} \equiv 5, \varphi_{23} \equiv 3, \varphi_{33} \equiv-1, \varphi_{32} \equiv-2, \varphi_{33} \equiv-5 ; \varphi_{11} \equiv 2, \varphi_{12} \equiv-1, \varphi_{13} \equiv 5, \varphi_{21} \equiv 4$, $\varphi_{22} \equiv 2, \varphi_{23} \equiv 1, \varphi_{33} \equiv-3, \varphi_{32} \equiv-4, \varphi_{33} \equiv 3$ and $\varphi_{11} \equiv-2, \varphi_{12} \equiv 1, \varphi_{13} \equiv-5, \varphi_{21} \equiv-4$, $\varphi_{22} \equiv-2, \varphi_{23} \equiv-1, \varphi_{33} \equiv 3, \varphi_{32} \equiv 4, \varphi_{33} \equiv-3$, respectively.

Remark 4.1 Since [4-9] only dealt with SICNNs without leakage delays, and [14-19, 23, 24] give no opinions about the problem of almost periodic solutions for SICNNs with time-varying leakage delays. One can observe that all the results in this literature and the references therein can not be applicable to prove the existence and exponential stability of almost periodic solutions for SICNNs (4.1). In this present paper, we employ a novel proof to establish some criteria to guarantee the existence and exponential stability of almost periodic solutions for SICNNs system with time-varying coefficients and leakage delays. What will happen when one can increase the neuron's order? Whether or not our results and method in this paper are available for this case, it is an interesting problem, and we leave it as our work in the future. 

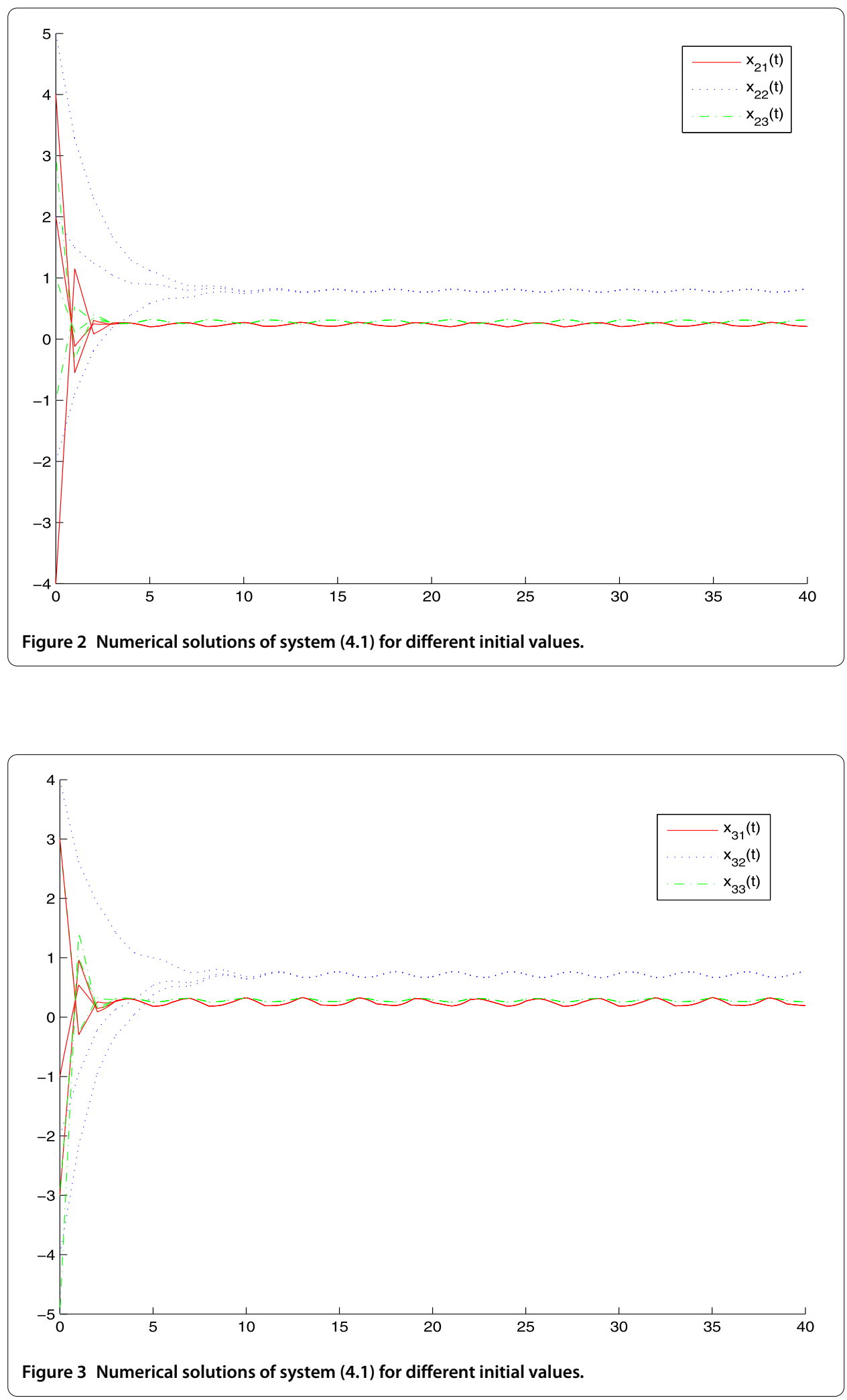


\section{Competing interests}

The authors declare that they have no competing interests.

\section{Authors' contributions}

BL gave the proof of Lemmas 2.1-2.2 and drafted the manuscript. JS proved Theorem 3.1 and gave the example to illustrate the effectiveness of the obtained results. All authors read and approved the final manuscript.

\section{Author details \\ ${ }^{1}$ College of Mathematics and Computer Science, Hunan University of Arts and Science, Changde, Hunan 415000, P.R. China. ${ }^{2}$ College of Mathematics, Physics and Information Engineering, Jiaxing University, Jiaxing, Zhejiang 314001, P.R. China.}

\section{Acknowledgements}

This work was supported by the construct program of the key discipline in Hunan province (Mechanical Design and Theory), the Scientific Research Fund of Hunan Provincial Natural Science Foundation of PR China (Grant No. 11 JJ6006), the Natural Scientific Research Fund of Hunan Provincial Education Department of PR China (Grants No. 11C0916, 11 C0915), and the Natural Scientific Research Fund of Zhejiang Provincial Education Department of PR China (Grants No. LY12A01018, Y6110436).

\section{Received: 23 March 2013 Accepted: 23 September 2013 Published: 07 Nov 2013}

\section{References}

1. Bouzerdoum, A, Pinter, RB: Shunting inhibitory cellular neural networks: derivation and stability analysis. IEEE Trans. Circuits Syst. I, Fundam. Theory Appl. 40, 215-221 (1993)

2. Bouzerdoum, A, Pinter, RB: Analysis and analog implementation of directionally sensitive shunting inhibitory cellular neural networks. In: Visual Information Processing: From Neurons to Chips. SPIE, vol. 1473, pp. $29-38$ (1991)

3. Bouzerdoum, A, Pinter, RB: Nonlinear lateral inhibition applied to motion detection in the fly visual system. In: Pinter, RB, Nabet, B (eds.) Nonlinear Vision, pp. 423-450. CRC Press, Boca Raton (1992)

4. Chen, A, Cao, J, Huang, L: Almost periodic solution of shunting inhibitory CNNs with delays. Phys. Lett. A 298, 161-170 (2002)

5. Chen, A, Huang, X: Almost periodic attractor of delayed neural networks with variable coefficients. Phys. Lett. A 340(1-4), 104-120 (2005)

6. Cai, M, Zhang, H, Yuan, Z: Positive almost periodic solutions for shunting inhibitory cellular neural networks with time-varying delays. Math. Comput. Simul. 78(4), 548-558 (2008)

7. Shao, J: Anti-periodic solutions for shunting inhibitory cellular neural networks with time-varying delays. Phys. Lett. A 372(30), 5011-5016 (2008)

8. Fan, Q, Shao, J: Positive almost periodic solutions for shunting inhibitory cellular neural networks with time-varying and continuously distributed delays. Commun. Nonlinear Sci. Numer. Simul. 15(6), 1655-1663 (2010)

9. Zhao, C, Fan, Q, Wang, W: Anti-periodic solutions for shunting inhibitory cellular neural networks with time-varying coefficients. Neural Process. Lett. 31, 259-267 (2010)

10. Kosko, B: Neural Networks and Fuzzy Systems. Prentice Hall, New Delhi (1992)

11. Haykin, S: Neural Networks. Prentice Hall, New Jersey (1999)

12. Gopalsamy, K: Stability and Oscillations in Delay Differential Equations of Population Dynamics. Kluwer Academic, Dordrecht (1992)

13. Gopalsamy, K: Leakage delays in BAM. J. Math. Anal. Appl. 325, 1117-1132 (2007)

14. Li, X, Cao, J: Delay-dependent stability of neural networks of neutral type with time delay in the leakage term. Nonlinearity 23, 1709-1726 (2010)

15. Li, X, Rakkiyappan, R, Balasubramaniam, P: Existence and global stability analysis of equilibrium of fuzzy cellular neural networks with time delay in the leakage term under impulsive perturbations. J. Franklin Inst. 348, 135-155 (2011)

16. Balasubramaniam, P, Vembarasan, V, Rakkiyappan, R: Leakage delays in T-S fuzzy cellular neural networks. Neural Process. Lett. 33, 111-136 (2011)

17. Liu, B: Global exponential stability for BAM neural networks with time-varying delays in the leakage terms. Nonlinear Anal., Real World Appl. 14, 559-566 (2013)

18. Chen, Z: A shunting inhibitory cellular neural network with leakage delays and continuously distributed delays of neutral type. Neural Comput. Appl. (2012). doi:10.1007/s00521-012-1200-2

19. Zhang, $\mathrm{H}$ : Global exponential stability of almost periodic solutions for SICNNs with continuously distributed leakage delays. Abstr. Appl. Anal. 2013, Article ID 307981 (2013). doi:10.1155/2013/307981

20. Fink, AM: Almost Periodic Differential Equations. Lecture Notes in Mathematics, vol. 377. Springer, Berlin (1974)

21. He, CY: Almost Periodic Differential Equation. Higher Education Publishing House, Beijing (1992) (in Chinese)

22. Hino, Y, Murakami, S, Nai, T: Functional Differential Equations with Infinite Delay. Lecture in Mathematics, vol. 1473. Springer, Berlin (1991)

23. Zhang, H, Shao, J: Almost periodic solutions for cellular neural networks with time-varying delays in leakage terms. Appl. Comput. Math. 219(24), 11471-11482 (2013)

24. Balasubramaniam, $P$, Vembarasan, V: Global asymptotic stability results for BAM neural networks of neutral-type with time delays in the leakage term under impulsive perturbations. Int. J. Comput. Math. 88, 3271-3291 (2011) 